\title{
Scalar-Flat Kähler Surfaces of All Genera
}

\author{
Jongsu Kim \\ ICTP, Trieste \\ Claude LeBrun \\ SUNY, Stony Brook \\ and \\ Massimiliano Pontecorvo \\ Università di Napoli
}

October 30, 2018

\begin{abstract}
Let $(M, J)$ be a compact complex 2-manifold which which admits a Kähler metric for which the integral of the scalar curvature is nonnegative. Also suppose that $M$ does not admit a Ricci-flat Kähler metric. Then if $M$ is blown up at sufficiently many points, the resulting complex surface $(\tilde{M}, \tilde{J})$ admits Kähler metrics with scalar curvature identically equal to zero. This proves Conjecture 1 of 16.
\end{abstract}

*Supported in part by NSF grant DMS 92-04093. 


\section{Introduction}

The problem of determining which compact complex manifolds $(M, J)$ admit Kähler metrics $g$ with constant scalar curvature was first formulated and studied by Calabi [5] in the late 1950's. Posed in this generality, Calabi's question is one to which the answer still eludes us; but there are two unrelated fronts on which notable progress has been made.

The most dramatic progress has been made in relation to the case in which one also requires that the Kähler class be a multiple of the manifold's first Chern class - that is, in relation to the question of which compact complex manifolds admit Kähler-Einstein metrics. This may be reformulated as an existence problem for solutions of the complex Monge-Ampère equation, and, working from this point of view, Aubin [2], Yau [30], and others [28, 25, 19, 27] have given us a fairly complete solution of the problem.

Progress, albeit of a more modest kind, has also been made regarding the case in which the scalar curvature of $g$ is required to equal zero and $\operatorname{dim}_{\mathbf{C}} M=$ 2 ; solutions $(M, J, g)$ of this problem are the scalar-flat Kähler surfaces of the title. The essential reason why this case is more tractable than others is that the underlying oriented Riemannian manifold $(M, g)$ of any scalar-flat Kähler surface is automatically [8] anti-self-dual, allowing one to invoke the Penrose twistor correspondence [20, 1] and giving rise to phenomena familiar from the theory of totally integrable systems. In particular, all scalar-flat Kähler surfaces with semi-free Killing fields can be written down explicitly [13, 14], and deforming these [16] leads to a reasonably complete picture when the fundamental group is large.

A fundamental limitation of the above approach is that any simplyconnected scalar-flat Kähler surface with a semi-free Killing field automatically must have large $\pi_{1}$, and solutions with small fundamental group are thus a priori inaccessible by this method. The present article, however, will finally prove the existence of (non-Ricci-flat) scalar-flat Kähler surfaces with $\pi_{1}=0$ and $\mathbf{Z} \oplus \mathbf{Z}$. Our key trick is a Kummer-type construction which allows us to produce new solutions by smoothing the orbifold singularities of $\mathbf{Z}_{2}$-quotients of old solutions. Successfully carrying this out involves melding 
two previous extensions [11, 17] of the Donaldson-Friedman method [6] of constructing anti-self-dual metrics on connected sums. After comparing the solutions obtained in this way with the biholomorphism types allowed by surface classification [29], we deduce the following:

Theorem A Let $(M, J)$ be a compact complex 2-manifold which admits a Kähler metric for which the integral of the scalar curvature is non-negative. Then precisely one of the following holds:

- $(M, J)$ admits a Ricci-flat Kähler metric; or

- any blow-up of $(M, J)$ has blow-ups $(\tilde{M}, \tilde{J})$ which admit scalar-flat Kähler metrics.

This proves Conjecture 1 of [16].

A corollary of this is the following:

Theorem B Let $(M, J)$ be a compact complex 2-manifold which admits a Kähler metric for which the integral of the scalar curvature is positive. Then any blow-up of $(M, J)$ has blow-ups $(\tilde{M}, \tilde{J})$ which admit Kähler metrics of constant positive scalar curvature.

One might note the formal similarity between Theorem A and a recent result of Taubes [26] which asserts that one can find anti-self-dual metrics on the connected sum of any smooth oriented 4-manifold $M$ with enough copies of $\overline{\mathbf{C P}}_{2}$. However, Taubes' proof is direct, whereas ours falls back on classification theory. One would hope that different proof of Theorem A, proceeding along Taubes' lines, might shed more light on Calabi's general problem.

\section{The Quotient Theorem}

In this section, we state the central technical result of this article, and then set up the framework in which it will be proved. This result, from which our main results will be deduced, is the following: 
Theorem 1 (Quotient Theorem) Let $\left(N, J_{N}, g_{N}\right)$ be a non-minimal compact complex surface with scalar-flat Kähler metric, and let $\Phi: N \rightarrow N$, $\Phi^{2}=1$, be a holomorphic isometry with only isolated fixed points. Let $\left(M, J_{M}\right)$ be obtained from $N / \Phi$ by replacing each singular point with a $\mathbf{C P}_{1}$ of self-intersection -2 . Then there exist scalar-flat Kähler metrics $g_{M}$ on $\left(M, J_{M}\right)$.

Here a compact complex surface $N$ is called non-minimal if is obtained from another surface by blowing up; this is equivalent to saying that $N$ contains a $\mathbf{C P}_{1}$ of self-intersection -1 . Our description of $M$ amounts to saying that if $N$ is blown up at the fixed points of $\Phi$, the resulting complex surface $\tilde{N}$ is a branched double cover of $M$, with the newly-introduced exceptional divisors as ramification locus. More abstractly, $M$ is the minimal resolution of the ordinary double-point singularities of the variety $N / \Phi$.

We will prove this theorem by using the theory of twistor spaces [1, 20]. For our purposes, a twistor space means a compact complex 3-manifold $Z$ equipped with a free anti-holomorphic involution $\sigma: Z \rightarrow Z$ and a foliation by $\sigma$-invariant rational curves $\mathbf{C P}_{1} \subset Z$ with normal bundle $\mathcal{O}(1) \oplus \mathcal{O}(1)$. Let $X$ denote the leaf space of this foliation by the so-called real twistor lines, and let $\wp: \tilde{Z} \rightarrow X$ denote the quotient map. There is then a canonical antiself-dual conformal metric $[g]$ on $X$, characterized by the requirement that the image of every holomorphic tangent space $T_{z}^{1,0} \tilde{Z}$ should be a $g$-isotropic subspace of $\mathbf{C} \otimes T X$. Conversely, every anti-self-dual manifold arises in this way, and does so in an essentially unique manner.

If a twistor space $Z$ contains a compact complex surface $D$ which is disjoint from its conjugate $\bar{D}:=\sigma(D)$ and has homological intersection number 1 with a twistor line, then $\left.\wp\right|_{D}: D \rightarrow X$ is a diffeomorphism, and $[g]$ pulls back from $X$ to yield a conformal class of Hermitian metrics on $D$. Because $D$ is compact, the anti-self-duality of $[g]$ implies [3, 21] that this conformal class is locally represented on $D$ by scalar-flat Kähler metrics. If, moreover, $b_{1}(D)$ is even, there is a globally-defined scalar-flat Kähler metric $g \in[g]$, and this global representative is uniquely determined once its total volume is specified; conversely, every scalar-flat Kähler surface arises from this construction, and does so in an essentially unique manner. Thus, in order to 
prove Theorem 11, it suffices to produce a twistor space $Z$ containing a copy of the complex surface $\left(M, J_{M}\right)$ which is disjoint from its conjugate and intersects some twistor line transversely in one point. We will do just this by refining the methods of [17], where anti-self-dual metrics were constructed on the underlying smooth manifold $M$.

To begin this construction, let $Z_{N}$ denote the twistor space of $\left(N, g_{N}\right)$, and let $L_{1}, \ldots, L_{k}$ be the twistor lines of the fixed points of $\Phi$. Let $\tilde{Z}_{N}$ be the blow-up of $Z_{N}$ along these lines, and let $Q_{1}, \ldots, Q_{k}$ be the exceptional divisors in $\tilde{Z}_{N}$ corresponding to $L_{1}, \ldots, L_{k}$; thus $Q_{j} \cong \mathbf{C P}_{1} \times \mathbf{C P}_{1}, j=1, \ldots k$, and each of these 2-quadrics has normal bundle $\mathcal{O}(1,-1) \rightarrow \mathbf{C P}_{1} \times \mathbf{C P}_{1}$. Since the derivative of $\Phi$ at its $k$ isolated fixed points must be -1 , the induced biholomorphism $\hat{\Phi}: \tilde{Z}_{N} \rightarrow \tilde{Z}_{N}$ fixes each $Q_{j}$ and acts on its normal bundle by -1 . The quotient $Z_{-}:=\tilde{Z}_{N} / \hat{\Phi}$ can thus be given the structure of a compact complex manifold in a unique way that the quotient map $\tilde{Z}_{N} \rightarrow Z_{-}$ becomes a branched covering, with $Q:=\cup Q_{j}$ as ramification locus. Let $Q_{j-}$ denote the image of this $Q_{j}$ in $Z_{-}$, which is an imbedded quadric with normal bundle $\mathcal{O}(2,-2)$, and let $Q_{-}=\bigcup Q_{j-}$.

The twistor space $Z_{N}$ contains a hypersurface $D_{N}$ corresponding to the complex structure $J_{N}$, as well as a disjoint hypersurface $\bar{D}_{N}:=\sigma\left(D_{N}\right)$ corresponding to the conjugate complex structure $-J_{N}$; indeed, $D_{N}$ and $\bar{D}_{N}$ are respectively isomorphic to $\left(N, \pm J_{N}\right)$ as complex surfaces. As the action of $\hat{\Phi}$ sends each such surface to itself, there are disjoint hypersurfaces $D_{-}$and $\bar{D}_{-}$ in $Z_{-}$obtained by first taking the proper transforms in $\tilde{Z}_{N}$ of $D_{N}$ and $\bar{D}_{N}$ and then projecting these hypersurfaces to $Z_{-}$. Notice that $D_{-}$is exactly a copy of $\left(M, J_{M}\right)$, whereas $\bar{D}_{-}$is a copy of $\left(M,-J_{M}\right)$. Set $\ell_{j-}:=D_{-} \cap Q_{j}$, $\bar{\ell}_{j-}:=\bar{D}_{-} \cap Q_{j}, \ell_{-}:=\bigcup \ell_{j-}$, and $\bar{\ell}_{-}:=\bigcup \bar{\ell}_{j-}$.

Our next step is to let $Z_{+}$consist of $k$ disjoint copies of the complex 3-fold $\tilde{Z}_{E H}$ obtained from the orbifold twistor space of the conformally compactified Eguchi-Hanson metric by blowing up the twistor line of infinity. To describe $\tilde{Z}_{E H}$ explicitly [10, 17], start with the $\mathbf{C P}_{3}$-bundle $\pi: \mathcal{B} \rightarrow \mathbf{C P}_{1}$ defined by

$$
\mathcal{B}=\mathbf{P}\left(\mathcal{O}(2)^{\oplus 3} \oplus \mathcal{O}\right)
$$

where our conventions are that $\mathbf{P}(E):=(E-0) / \mathbf{C}^{\times}$. Let $\mathcal{O}(1,0):=\pi^{*} \mathcal{O}(1)$, and let $\mathcal{O}(0,-1)$ be the universal bundle, whose principal $\mathbf{C}^{\times}$-bundle is 
$\left[\left(\mathcal{O}(2)^{\oplus 3} \oplus \mathcal{O}\right)-0\right] \rightarrow \mathcal{B}$. The "homogeneous coordinates" of $\mathcal{O}(2)^{\oplus 3} \oplus \mathcal{O}$ are canonical sections $x, y, z \in \Gamma \mathcal{O}(2,1)$ and $t \in \Gamma \mathcal{O}(0,1)$. Let $a \in \Gamma\left(\mathbf{C P}_{1}, \mathcal{O}(2)\right)$ be a non-trivial section which is invariant under the anti-holomorphic involution $\mathbf{j}$ of $\mathcal{O}(2)=T \mathbf{C P}_{1}$ induced by the antipodal map of $S^{2}=\mathbf{C P}_{1}$; and let the 2 distinct zeroes of $a$ be called the north and south poles. Our blown-up twistor space is then obtained from the hypersurface

$$
x y=z^{2}-t^{2} a^{2}
$$

in $\mathcal{B}$ by replacing the two singular points $x=y=z=0$ with $\mathbf{C P}_{1}$ 's. The quadric $t=0$ corresponds to the blow-up of the orbifold twistor line "at infinity," whereas the real structure is given by

$$
[x: y: z: t] \rightarrow[\mathbf{j}(y): \mathbf{j}(x): \mathbf{j}(z): \bar{t}]
$$

If we let $D_{E H} \subset \tilde{Z}_{E H}$ denote the Hirzebruch surface over the south pole and let $\bar{D}_{E H}$ denote the Hirzebruch surface over the north pole, then we may define $D_{+} \subset Z_{+}$and $\bar{D}_{+} \subset Z_{+}$to consist of $k$ disjoint copies of $D_{E H}$ and $\bar{D}_{E H}$, respectively. Let us use $Q_{j+}$ to denote the appropriate copy of the $t=0$ quadric in $\tilde{Z}_{E H}$ and $Q_{+}$to denote $\cup Q_{j+}$. Set $\ell_{j+}=Q_{j+} \cap D_{+}$, $\bar{\ell}_{j+}=Q_{j+} \cap \bar{D}_{+}, \ell_{+}=\bigcup \ell_{j+}$, and $\bar{\ell}_{+}=\bigcup \bar{\ell}_{j+}$.

Now let $Z_{0}=Z_{-} \cup_{Q_{j}} Z_{+}$be obtained from the disjoint union $Z_{-} \sqcup Z_{+}$ by biholomorphically identifying $Q_{j-} \subset Z_{-}$with $Q_{j+} \subset Z_{+}$in such a way that the real structures agree and such that $\ell_{j-}$ is identified with $\ell_{j+}$. (This actually specifies the gluing procedure uniquely, modulo real automorphisms of $Z_{+}$.) Set $D_{0}=D_{-} \cup D_{+}$and $\bar{D}_{0}=\bar{D}_{-} \cup \bar{D}_{+}$. The result is that $Z_{0}, D_{0}$ and $\bar{D}_{0}$ are complex spaces with normal crossing singularities, and there is an induced real structure $\sigma: Z_{0} \rightarrow Z_{0}$ which interchanges $D_{0}$ and $\bar{D}_{0}$. By a slight abuse of notation, we will denote the image of $Q_{j \pm}$ in $Z_{0}$ by $Q_{j}$, whereas the images of $\ell_{j \pm}, \bar{\ell}_{j \pm}, \ell_{ \pm}, \bar{\ell}_{ \pm}$, and $Q_{ \pm}$will respectively be denoted by $\ell_{j}, \bar{\ell}_{j}, \ell, \bar{\ell}$, and $Q$.

Our method of proving Theorem 11 will now be as follows: we will construct twistor spaces $Z_{t}$ containing hypersurfaces $D_{t} \cong\left(M, J_{M}\right)$ by simultaneously smoothing the singularities of $Z_{0}$ and $D_{0} \subset Z_{0}$. 


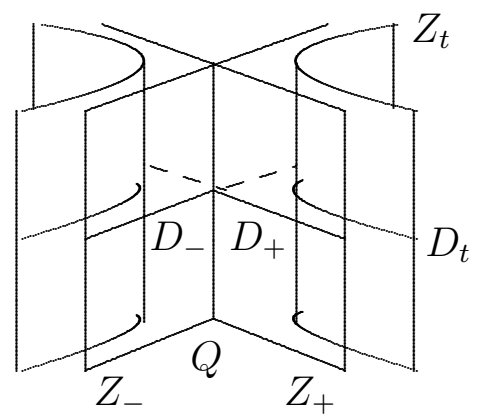

Because the twistor spaces $Z_{t}$ must admit real structures, we will of course also need to smooth the singularities of $\bar{D}_{0}$, too, and it is natural to also include this stipulation from the outset. In $\S 3$, Theorem 1 will now be proved constructing relative smoothings of precisely this type.

\section{Proof of the Quotient Theorem}

Continuing the discussion of $\$ 2$, let $D_{0} \bar{D}_{0} \subset Z_{0}$ denote the disjoint union of $D_{0}$ and $\bar{D}_{0}=\sigma\left(D_{0}\right)$, and let $f: D_{0} \bar{D}_{0} \hookrightarrow Z_{0}$ be the tautological holomorphic imbedding. In this section we shall prove Theorem 11 by studying the deformation theory of the pair of the pair $\left(Z_{0}, D_{0} \bar{D}_{0}\right)$. Our approach depends crucially upon the results of Ran [22, 23].

Let us first warm up by discussing deformations of the singular surface $D_{0}$, noting all along that such a discussion will, by conjugation, automatically also implicitly completely describe the deformation theory of $\bar{D}_{0}$, and hence that of the disjoint union $D_{0} \bar{D}_{0}=D_{0} \sqcup \bar{D}_{0}$, too. Now since $D_{0}=D_{-} \cup_{l} D_{+}$is obtained from $D_{-} \sqcup D_{+}$by identifying $(-2)$-curves $\ell_{j-} \subset D_{-}$with $(+2)$-curves $\ell_{j+} \subset D_{+}, D_{0}$ is a singular complex surface with normal crossing singularities

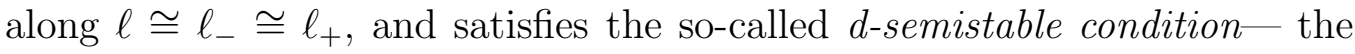
two normal bundles $\nu_{\ell_{ \pm}, D_{ \pm}}$of the singular hypersurface are dual to each other.

Because $D_{+}$has $k$ connected components, each of which is isomorphic to the second Hirzebruch surface $\mathbf{P}(\mathcal{O} \oplus \mathcal{O}(2))$, and as each connected component of $\ell_{+}$corresponds to the zero section of $\mathcal{O}(2) \subset \mathbf{P}(\mathcal{O} \oplus \mathcal{O}(2))$, it follows that

$$
h^{j}\left(\Theta_{D_{+}} \otimes \mathcal{I}_{\ell_{+}}\right)=0 \quad \forall j,
$$




$$
h^{j}\left(\Theta_{D_{+}}\right)= \begin{cases}6 k & j=0 \\ 0 & j \neq 0\end{cases}
$$

and

$$
h^{j}\left(\Theta_{D_{+}, \ell_{+}}\right)=h^{j}\left(\Theta_{\ell_{+}}\right)=h^{j}\left(\nu_{\ell_{+}, D_{+}}\right)=\left\{\begin{array}{ll}
3 k & j=0 \\
0 & j \neq 0
\end{array},\right.
$$

where $\Theta_{Y, X}$ denotes the sheaf of holomorphic vector fields on $Y$ which represent 0 in the normal bundle $\nu_{X, Y}$ of the complex submanifold $X \subset Y$. Using these facts, we may now prove the following:

Lemma 1 Let $\tau_{D_{0}}^{0}$ denote the sheaf of derivations of $\mathcal{O}_{D_{0}}$. Then

$$
H^{j}\left(\tau_{D_{0}}^{0}\right) \cong \begin{cases}H^{0}\left(\Theta_{D_{-}}\right) & j=0 \\ H^{1}\left(\Theta_{D_{-}, \ell_{-}}\right) & j=1 \\ 0 & j \geq 2 .\end{cases}
$$

Proof. Consider the normalization exact sequence

$$
0 \longrightarrow \tau_{D_{0}}^{0} \longrightarrow q_{*} \Theta_{D_{-} \sqcup D_{+}, \ell_{-} \sqcup \ell_{+}} \longrightarrow \iota_{*} \Theta_{\ell} \longrightarrow 0,
$$

where $q: D_{-} \sqcup D_{+} \rightarrow D_{0}$ is the quotient map and $\iota: \ell \rightarrow D_{0}$ is the inclusion. Since the restriction map $H^{0}\left(\Theta_{D_{+}, \ell_{+}}\right) \rightarrow H^{0}\left(\Theta_{\ell}\right)$ is an isomorphism, the associated long exact sequence tells us that $H^{0}\left(\tau_{D_{0}}^{0}\right)=H^{0}\left(\Theta_{D_{-}, \ell_{-}}\right)=H^{0}\left(\Theta_{D_{-}}\right)$, whereas $H^{1}\left(\tau_{D_{0}}^{0}\right)=H^{1}\left(\Theta_{D_{-}, \ell_{-}}\right)$.

Since $H^{2}\left(\Theta_{D_{+}, \ell_{+}}\right)=0$, we also read off that $H^{2}\left(\tau_{D_{0}}^{0}\right)=H^{2}\left(\Theta_{D_{-}, \ell_{-}}\right)$, and it only remains for us to show that the latter cohomology group vanishes. But $D_{-}$is a ruled surface, and the generic $\mathbf{C P}_{1}$ fiber of $D_{-}$is disjoint from $\ell_{-}$; indeed, $N$ is ruled because 29 it is non-minimal and admits a scalar-flat Kähler metric, $\tilde{N}$ is obtained from $N$ by blowing up, and $\tilde{N}$ is a branched cover of $D_{-} \cong M$, with branch locus $\ell_{-}$the union of the exceptional divisors introduced by the blow-up $\tilde{N} \rightarrow N$. Moreover, $\Theta_{D_{-}, \ell_{-}}$is a locally free sheaf, and Serre duality therefore says that $H^{2}\left(D_{-}, \Theta_{D_{-}, \ell_{-}}\right)=$ $\left[H^{0}\left(D_{-}, H o m\left(\Theta_{D_{-}, \ell_{-}}, \Omega^{2}\right)\right)\right]^{*}$. The restriction of $\operatorname{Hom}\left(\Theta_{D_{-}, \ell_{-}}, \Omega_{D_{-}}^{2}\right)$ to a generic $\mathbf{C P}_{1}$ fiber of $D_{-}$is thus isomorphic to $\mathcal{O}(-2) \oplus \mathcal{O}(-4)$, and any global section of this sheaf therefore vanishes. Hence $H^{2}\left(\tau_{D_{0}}^{0}\right)=H^{2}\left(D_{-}, \Theta_{D_{-}, \ell_{-}}\right)=$ 0 , as claimed.

This implies that the deformation theory of $D_{0}$ is unobstructed: 
Proposition 1 The complex space $D_{0}$ admits a versal deformation

$$
\mathcal{D} \stackrel{\varpi}{\longrightarrow} H^{1}\left(D_{-}, \Theta_{D_{-}, \ell_{-}}\right) \times \mathbf{C}^{k},
$$

with fibers $D_{t}:=\varpi^{-1}\left(t_{1}, t_{2}\right), t_{1} \in H^{1}\left(D_{-}, \Theta_{D_{-}, \ell_{-}}\right), t_{2} \in \mathbf{C}^{k}$, satisfying

1. $D_{t}$ is smooth iff $t_{2} \in\left(\mathbf{C}^{\times}\right)^{k}$;

2. $D_{t} \cong D_{-}=M$ when $t_{1}=0$ and $t_{2} \in\left(\mathbf{C}^{\times}\right)^{k}$; and

3. all small deformations of $D_{-}=M$ occur as smooth fibers $D_{t}$ of $\varpi$.

Proof. The deformation theory [7, [6] of $D_{0}$ is governed by the vector spaces $T_{D_{0}}^{j}=\operatorname{Ext}^{j}\left(\Omega_{D_{0}}^{1}, \mathcal{O}_{D_{0}}\right)$. These may be computed by means of the Ext spectral sequence [9]

$$
E_{2}^{p, q}=H^{p}\left(D_{0}, \tau_{D_{0}}^{j}\right) \Longrightarrow T_{D_{0}}^{p+q},
$$

where $\tau_{D_{0}}^{j}=E x t^{j}\left(\Omega^{1}, \mathcal{O}\right)$. Because $D_{0}$ is a locally complete intersection, $\tau_{D_{0}}^{j}=0$ for $j \geq 2$, and the spectral sequence therefore degenerates into the exact sequences

$$
H^{j-2}\left(\tau_{D_{0}}^{1}\right) \rightarrow H^{j}\left(\tau_{D_{0}}^{0}\right) \rightarrow T_{D_{0}}^{j} \rightarrow H^{j-1}\left(\tau_{D_{0}}^{1}\right) \rightarrow H^{j+1}\left(\tau_{D_{0}}^{0}\right) .
$$

Meanwhile, the d-semi-stable condition tells us tells us that $\tau_{D_{0}}^{0} \cong \mathcal{O}_{\ell}$, so

$$
H^{j}\left(\tau_{D_{0}}^{1}\right) \cong \begin{cases}\mathbf{C}^{k} & j=0 \\ 0 & j \neq 0\end{cases}
$$

The lemma now tells us that $T_{D_{0}}^{2}=0$, and that there is an exact sequence

$$
0 \rightarrow H^{1}\left(D_{-}, \Theta_{D_{-}, \ell_{-}}\right) \rightarrow T_{D_{0}}^{1} \rightarrow \mathbf{C}^{k} \rightarrow 0 .
$$

Since $T_{D_{0}}^{2}=0$, the deformation theory [7] of $D_{0}$ is unobstructed, and there is a versal family $\mathcal{D}$ over a neighborhood of $0 \in T_{D_{0}}^{1}$. This family has the property that any 1-dimensional subfamily of this family smooths the normal crossing at $\ell_{j}$ if the image of its derivative in $H^{0}\left(\tau_{\ell}^{1}\right) \cong H^{0}(\ell, \mathcal{O})=\mathbf{C}^{k}$ is 
non-zero on $\ell_{j}$. Moreover, an effectively parametrized family with central fiber $D_{0}$ is a subfamily of $\mathcal{D}$.

Let us now consider the explicit smoothing $\mathcal{M}$ of $D_{0}$ gotten by blowing up $M \times \mathbf{C}^{k}$ along the smooth submanifold $\left(\ell_{1} \times\left\{a_{1}=0\right\}\right) \cup \cdots \cup\left(\ell_{k} \times\left\{a_{k}=0\right\}\right)$. Since $\ell_{j} \subset M$ has self-intersection -2 , the central fiber of $\mathcal{M} \rightarrow \mathbf{C}^{k}$ is obtained from $D_{-}=M$ by attaching a Hirzebruch surface $\mathbf{P}(\mathcal{O}(-2) \oplus \mathcal{O})$ to $M$ at each $\ell_{j}$; in other words, the central fiber is isomorphic to $D_{0}$. Since $\mathcal{M}$ is an effectively parametrized family with central fiber $D_{0}$, it must be contained in $\mathcal{D}$ by versality, and because the fiber of $\mathcal{M}$ over any $t_{2} \in\left(\mathbf{C}^{\times}\right)^{k}$ is smooth, the image of $\mathbf{C}^{k}$ in $T_{D_{0}}^{1}$ is transverse to the kernel of $T_{D_{0}}^{1} \rightarrow$ $H^{0}\left(\tau_{\ell}^{1}\right) \cong \mathbf{C}^{k}$. We may now choose new coordinates on $T_{D_{0}}^{1}$, identifying it with $H^{1}\left(D_{-}, \Theta_{D_{-}, \ell_{-}}\right) \times \mathbf{C}^{k}$ in such a way that the above explicit family corresponds to the $\mathbf{C}^{k}$ subspace, and so that the $H^{1}\left(D_{-}, \Theta_{D_{-}, \ell_{-}}\right)$subspace corresponds to the original kernel of the projection.

If $\mathbf{M} \rightarrow \Delta$ is any deformation of $M$ over a neighborhood of $0 \in \mathbf{C}$, its blow-up $\tilde{\mathbf{M}}$ at the submanifold $\ell_{-}$of the central fiber is a smoothing of $D_{0}$. By versality, $\tilde{\mathbf{M}}$ must therefore be a subfamily of $\mathcal{D}$, amd every small deformation of $M$ thus occurs as a smooth fiber $D_{t}$.

Remark. At the tangent space level, the above proof shows that the exact sequence

$$
0 \rightarrow H^{1}\left(\tau_{D_{0}}^{0}\right) \rightarrow T_{D_{0}}^{1} \rightarrow H^{0}\left(\tau_{D_{0}}^{1}\right) \rightarrow 0
$$

has a geometrically preferred splitting. This will later prove useful.

Having discussed the deformation theory of $D_{0} \bar{D}_{0}$, the next step is obviously to discuss that of $Z_{0}$; but this, in fact, has already been studied in [17]. As before, the vector spaces $T_{Z_{0}}^{q}=\operatorname{Ext}^{q}\left(\Omega_{Z_{0}}^{1}, \mathcal{O}_{Z_{0}}\right)$ that control the deformation theory fit into an exact sequence

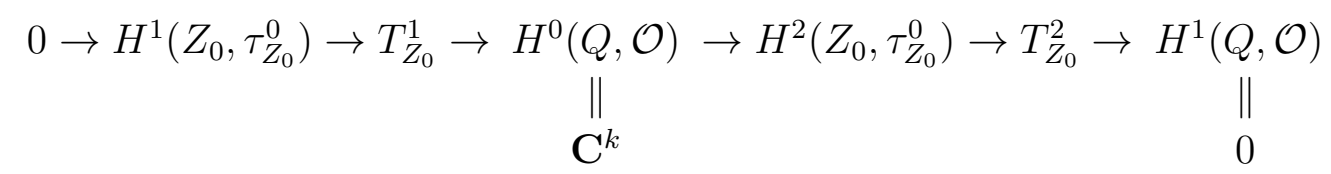


and the cohomology of $\tau_{Z_{0}}^{0}=\mathcal{H o m}\left(\Omega_{Z_{0}}^{1}, \mathcal{O}_{Z_{0}}\right)$ can in turn be computed via the long exact sequence

$H^{j-1}\left(Q, \Theta_{Q}\right) \rightarrow H^{j}\left(Z_{0}, \tau_{Z_{0}}^{0}\right) \rightarrow H^{j}\left(Z_{-}, \Theta_{Z_{-}, Q}\right) \oplus H^{j}\left(Z_{+}, \Theta_{Z_{+}, Q}\right) \rightarrow H^{j}\left(Q, \Theta_{Q}\right)$

Using the explicit form of $Z_{+}$, one then may check that $H^{2}\left(Z_{+}, \Theta_{Z_{+}, Q}\right)=0$. On the other hand, $Z_{-}$is has a branched cover which is a blow-up of the twistor space $Z_{N}$, and this implies that $H^{2}\left(Z_{-}, \Theta_{Z_{-}, Q}\right)=\left[H^{2}\left(Z_{N}, \Theta_{Z_{-}, Q}\right)\right]_{\Phi}$, where the subscript indicates the +1 -eigenspace of the automorphism induced by $\Phi$. One may thus conclude that $T_{Z_{0}}^{2}=0$, and that the smoothing theory of $Z_{0}$ is therefore unobstructed, once one knows the following result, which was stated in [16]:

Theorem 2 Suppose that $N$ is a non-minimal compact complex surface with scalar-flat Kähler metric $g_{N}$. Then its twistor space satisfies $H^{2}\left(Z_{N}, \Theta\right)=$ $H^{2}\left(Z_{N}, \Theta \otimes \kappa^{-1 / 2}\right)=H^{2}\left(Z_{N}, \Theta_{Z, D \bar{D}}\right)=0$, where $D$ and $\bar{D}$ are canonical divisors associated with $J_{N}$ and $-J_{N}$.

Unfortunately, while the proof given in [16] suffices for all cases needed for our applications, it overlooks the case of surfaces with non-semi-free $\mathbf{C}^{\times}$actions, and we have therefore chosen to include a completed proof in the present article. As this proof is rather long, however, and involves ideas quite unrelated to the thrust of the present discussion, it has been relegated to an appendix $(\$ 5)$.

Finally, we turn to the deformation theory of the pair $\left(Z_{0}, D_{0} \bar{D}_{0}\right)$, which is governed by the derived functors $T_{f}^{j}$ of Ran [22] [23], in the sense that $T_{f}^{1}$ corresponds to infinitesimal deformations of the imbedding $f$ and obstructions lie in $T_{f}^{2}$. These vector spaces may be computed by means of a long exact sequence

$$
\begin{aligned}
0 & \longrightarrow T_{f}^{0} \longrightarrow T_{D_{0} \bar{D}_{0}}^{0} \oplus T_{Z_{0}}^{0} \longrightarrow \operatorname{Ext}_{f}^{0}\left(\Omega_{Z_{0}}^{1}, \mathcal{O}_{D_{0} \bar{D}_{0}}\right) \\
& \longrightarrow T_{f}^{1} \longrightarrow T_{D_{0} \bar{D}_{0}}^{1} \oplus T_{Z_{0}}^{1} \longrightarrow \operatorname{Ext}_{f}^{1}\left(\Omega_{Z_{0}}^{1}, \mathcal{O}_{D_{0} \bar{D}_{0}}\right) \\
& \longrightarrow T_{f}^{2} \longrightarrow T_{D_{0} \bar{D}_{0}}^{2} \oplus T_{Z_{0}}^{2} \longrightarrow \cdots
\end{aligned}
$$


Here $T_{D_{0} \bar{D}_{0}}^{j}:=\operatorname{Ext}^{j}\left(\Omega_{D_{0}}^{1}, \mathcal{O}_{D_{0}}\right) \oplus \operatorname{Ext}^{j}\left(\Omega_{\bar{D}_{0}}^{1}, \mathcal{O}_{\bar{D}_{0}}\right)$ and $T_{Z_{0}}^{j}:=\operatorname{Ext}^{j}\left(\Omega_{Z_{0}}^{1}, \mathcal{O}_{Z_{0}}\right)$ are the usual global Ext groups, whereas $\operatorname{Ext}_{f}^{j}\left(\Omega_{Z_{0}}^{1}, \mathcal{O}_{D_{0} \bar{D}_{0}}\right)$ are the derived functors of

$$
\operatorname{Hom}_{f}\left(\Omega_{Z_{0}}^{1}, \mathcal{O}_{D_{0} \bar{D}_{0}}\right):=\operatorname{Hom}_{\mathcal{O}_{D_{0} \bar{D}_{0}}}\left(f^{*} \Omega_{Z_{0}}^{1}, \mathcal{O}_{D_{0} \bar{D}_{0}}\right) \cong \operatorname{Hom}_{\mathcal{O}_{Z_{0}}}\left(\Omega_{Z_{0}}^{1}, f_{*} \mathcal{O}_{D_{0} \bar{D}_{0}}\right)
$$

in either variable.

The local form of the singularities of the pair $\left(Z_{0}, D_{0} \bar{D}_{0}\right)$ is just the same as in [11, §3]. Namely, around the singular locus we can take coordinates $\left(w_{1}, \ldots, w_{4}\right) \in \mathbf{C}^{4}$ so that $Z_{0}$ is given by $\left\{w_{1} w_{2}=0\right\}$ and $D_{0}$ (or $\left.\bar{D}_{0}\right)$ is the hypersurface $\left\{w_{4}=0\right\}$. Because of this we have a spectral sequence

$$
E_{2}^{p, q}=H^{p}\left(\mathcal{E} x t^{p}\left(\Omega_{Z_{0 \mid D_{0} \bar{D}_{0}}}^{1}, \mathcal{O}_{D_{0} \bar{D}_{0}}\right)\right) \Rightarrow \operatorname{Ext}_{f}^{p+q}\left(\Omega_{Z_{0}}^{1}, \mathcal{O}_{D_{0} \bar{D}_{0}}\right)
$$

where the local $\mathcal{E} x t$ sheaves can be computed [11, 3.10] to be

$$
\mathcal{E} x t^{r}\left(\left.\Omega_{Z_{0}}^{1}\right|_{D_{0} \bar{D}_{0}}, \mathcal{O}_{D_{0} \bar{D}_{0}}\right) \cong \begin{cases}\left.\tau_{Z_{0}}^{0}\right|_{D_{0} \bar{D}_{0}}, & r=0 \\ \tau_{D_{0} \bar{D}_{0}}^{1} \cong \mathcal{O}_{\ell \bar{\ell}}, & r=1 \\ 0, & r \geq 2\end{cases}
$$

We now claim that the relative deformation theory under consideration is unobstructed as a consequence of Theorem 2. As in [11, §4], we start by considering the exact sequence

$$
\left.0 \longrightarrow \tau_{Z_{0}, D_{0} \bar{D}_{0}}^{0} \longrightarrow \tau_{D_{0} \bar{D}_{0}}^{0} \oplus \tau_{Z_{0}}^{0} \longrightarrow \tau_{Z_{0}}^{0}\right|_{D_{0} \bar{D}_{0}} \longrightarrow 0 .
$$

Lemma 2 Suppose that $N$ satisfies the hypothesis of the Quotient Theorem. Then $H^{2}\left(\tau_{Z_{0}, D_{0} \bar{D}_{0}}^{0}\right)=H^{2}\left(\left.\tau_{Z_{0}}^{0}\right|_{D_{0} \bar{D}_{0}}\right)=0$.

Proof. Since we have already observed that $H^{2}\left(\tau_{D_{0} \bar{D}_{0}}^{0}\right) \oplus H^{2}\left(\tau_{Z_{0}}^{0}\right)=0$, it is enough to show that $H^{j}\left(\tau_{Z_{0}, D_{0} \bar{D}_{0}}^{0}\right)$ for $j=2,3$. To this end, consider the exact normalization sequence

$$
0 \longrightarrow \tau_{Z_{0}, D_{0} \bar{D}_{0}}^{0} \longrightarrow \Theta_{Z_{+}, D_{+} \bar{D}_{+} Q} \oplus \Theta_{Z_{-}, D_{-} \bar{D}_{-} Q_{-}} \longrightarrow \Theta_{Q, \ell \bar{\ell}} \longrightarrow 0
$$


where $\ell=Q_{-} \cap D_{-}=Q_{-} \cap D_{+}$and $\bar{\ell}=Q_{-} \cap \bar{D}_{-}=Q \cap \bar{D}_{+}$. Because

$$
0 \longrightarrow \Theta_{Q, \ell \bar{\ell}} \longrightarrow \Theta_{Q} \longrightarrow \nu_{\ell} \oplus \nu_{\bar{\ell}} \longrightarrow 0
$$

is exact and $H^{0}\left(\Theta_{Q}\right) \rightarrow H^{0}\left(\nu_{\ell} \oplus \nu_{\bar{\ell}}\right)$ is surjective, it follows that

$$
H^{j}\left(\tau_{Z_{0}, D_{0} \bar{D}_{0}}^{0}\right)=H^{j}\left(\Theta_{Z_{+}, D_{+} \bar{D}_{+} Q}\right) \oplus H^{j}\left(\Theta_{Z_{-}, D_{-} \bar{D}_{-} Q_{-}}\right), \quad j=2,3 .
$$

But rational curves of normal bundle $\mathcal{O}(1) \oplus \mathcal{O}(1)$ sweep out an open subset of $Z_{ \pm}$, so Serre duality tells us that $H^{3}\left(\Theta_{Z_{ \pm}, D_{ \pm} \bar{D}_{ \pm} Q_{ \pm}}\right)=0$. It thus only remains to show that $H^{2}\left(\Theta_{Z_{ \pm}, D_{ \pm} \bar{D}_{ \pm} Q_{ \pm}}\right)=0$.

We start by considering $Z_{-}$, which, by construction, fits into a diagram

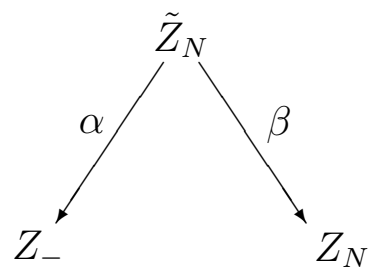

in which $\beta$ blows a disjoint union $Q$ of quadrics down into the union $L_{\Phi}$ of $\Phi$-invariant twistor lines in $Z_{N}$, and in which $\alpha$ is a 2-fold branched covering map with branch locus $Q$. Let $\mathcal{L}$ be the divisor square-root of $Q \subset Z_{N}$ which is associated with this branched cover. Then

$$
\alpha_{*}^{j} \Theta_{\tilde{Z}_{N}, \tilde{D}_{N} \tilde{\bar{D}}_{N} Q}= \begin{cases}\Theta_{Z_{-}, D_{-} \bar{D}_{-} Q_{-}} \oplus\left(\Theta_{Z_{-}, D_{-} \bar{D}_{-} Q_{-}} \otimes \mathcal{L}\right) & j=0 \\ 0 & j \neq 0\end{cases}
$$

whereas

$$
\beta_{*}^{j} \Theta_{\tilde{Z}_{N}, \tilde{D}_{N} \tilde{\bar{D}}_{N} Q}= \begin{cases}\Theta_{Z_{N}, D_{N} \bar{D}_{N}, L_{\Phi}} & j=0 \\ 0 & j \neq 0 .\end{cases}
$$

In combination with the short exact sequence

$$
0 \longrightarrow \Theta_{Z_{N}, D_{N} \bar{D}_{N}, L_{\Phi}} \longrightarrow \Theta_{Z_{N}, D_{N} \bar{D}_{N}} \longrightarrow \nu_{L_{\Phi}, Z_{N}} \longrightarrow 0
$$

and the observation that $\nu_{L_{\Phi}, Z_{N}} \cong \mathcal{O}(1) \oplus \mathcal{O}(1)$ on each $\mathbf{C P}_{1}$ component, (3.3) tells us that

$$
H^{2}\left(\tilde{Z}_{N}, \Theta_{\tilde{Z}_{N}, \tilde{D}_{N} \tilde{\bar{D}}_{N} Q}\right)=H^{2}\left(Z_{N}, \Theta_{Z_{N}, D_{N} \bar{D}_{N}, L_{\Phi}}\right)=H^{2}\left(Z_{N}, \Theta_{Z_{N}, D_{N} \bar{D}_{N}}\right)=0 .
$$


But the Leray spectral sequence of (3.2) says us that

$$
\left[H^{2}\left(\tilde{Z}_{N}, \Theta_{\tilde{Z_{N}}, \tilde{D}_{N} \tilde{\bar{D}}_{N} Q}\right)\right]_{\Phi} \cong H^{2}\left(Z_{-}, \Theta_{Z_{-}, D_{-} \bar{D}_{-} Q_{-}}\right)
$$

so that $H^{2}\left(Z_{-}, \Theta_{Z_{-}, D_{-} \bar{D}_{-} Q}\right)=0$, as claimed.

To finish the proof we have to show $H^{2}\left(Z_{+}, \Theta_{Z_{+}, D_{+} \bar{D}_{+} Q}\right)=0$. But 17, Lemma 2] says that $H^{2}\left(Z_{+}, \Theta_{Z_{+}, Q_{+}}\right)=0$. We now invoke the exact sequence

$$
0 \longrightarrow \Theta_{Z_{+}, D_{+} \bar{D}_{+} Q_{+}} \longrightarrow \Theta_{Z_{+}, Q} \longrightarrow \nu_{D_{+} \bar{D}_{+}, Z_{+}} \longrightarrow 0
$$

But because $\nu_{D_{+} \bar{D}_{+}, Z_{+}}$is trivial, it follows that $H^{1}\left(\nu_{D_{+} \bar{D}_{+}, Z_{+}}\right)=H^{1}\left(D_{+} \bar{D}_{+}, \mathcal{O}\right)=$ 0 , since each component of $D_{+} \bar{D}_{+}$is a simply-connected surface. This implies that $H^{2}\left(Z_{+}, \Theta_{Z_{+}, D_{+} \bar{D}_{+} Q}\right)=H^{2}\left(Z_{+}, \Theta_{Z_{+}, Q}\right)=0$, as desired.

As a consequence, we have a commutative diagram

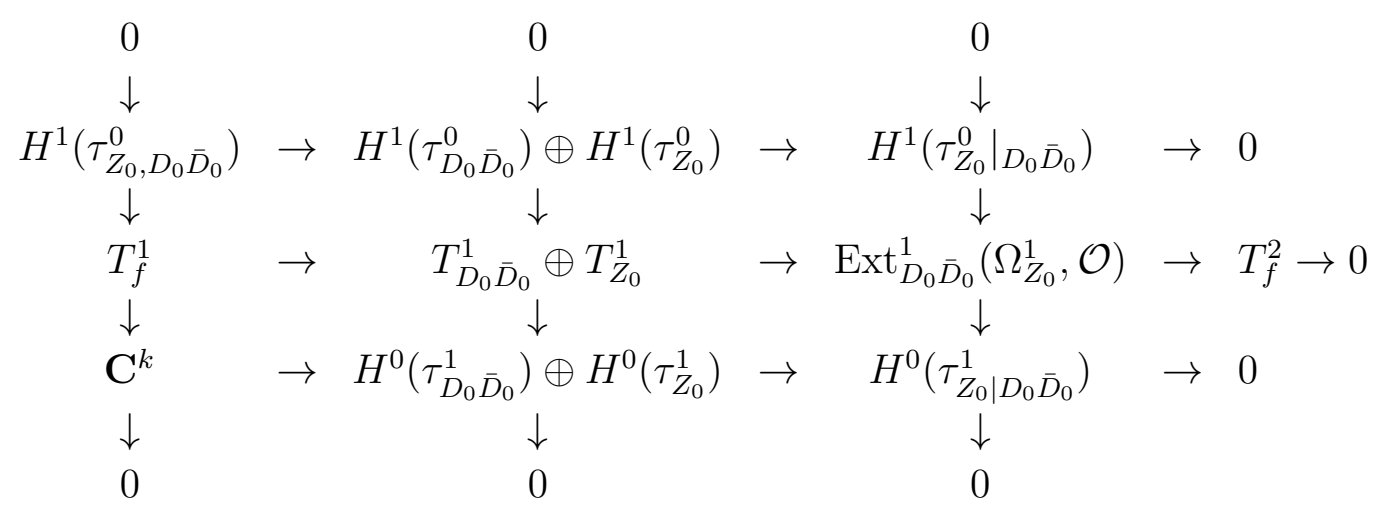

with exact rows and columns, and where the middle row is (3.1). In particular, $T_{f}^{2}=0$, so there exists a versal deformation

$$
\begin{aligned}
\mathcal{D} \sqcup \overline{\mathcal{D}} \hookrightarrow & \mathcal{Z} \\
\varpi_{D} \searrow & \downarrow_{\varpi_{Z}} \\
& U \stackrel{\text { open }}{\subset} T_{f}^{1}
\end{aligned}
$$

such that the fibers of $\varpi_{Z}$ and $\varpi_{D}$ are smooth over elements of $T_{f}^{1}$ which project to $\left(\mathbf{C}^{\times}\right)^{k} \subset \mathbf{C}^{k}$. Furthermore, by the same argument as [6, $\S 6.1$ ], there is a real structure $\hat{\sigma}: \mathcal{Z} \rightarrow \mathcal{Z}$ which interchanges $\mathcal{D}$ and $\overline{\mathcal{D}}$ and which 
restricts to the central fiber as the given real structure $\sigma$ on $Z_{0}$. This induces a complex conjugation on $T_{f}^{1}$ compatible with the standard one on $\mathbf{C}^{k}$, and the fibers $Z_{t}$ over points $t$ of the real slice which project to $\left(\mathbf{R}^{\times}\right)^{k} \subset \mathbf{R}^{k}$ are twistor spaces [6, 17]. Moreover, these twistor spaces contain degree-1 divisors $D_{t}$ which are disjoint from their images $\bar{D}_{t}$ under the real structure; and since $b_{1}\left(D_{t}\right)=b_{1}(M)$ is even, it therefore follows [3, 21] that any such $Z_{t}$ is the twistor space of of a scalar-flat Kähler metric on $D_{t}$.

To prove the Quotient Theorem, it thus suffices to show that there are suitable real values of $t \in T_{f}$ for which $D_{t}$ is biholomorphic to $M$. In order to show that this is possible, we will use the following:

Lemma 3 For $N$ as above, the natural map

$$
H^{1}\left(\tau_{Z_{0}, D_{0} \bar{D}_{0}}^{0}\right) \rightarrow H^{1}\left(\tau_{D_{0} \bar{D}_{0}}^{0}\right)
$$

is a surjection.

Proof. The same normalization sequence of the preceeding lemma tells us that the natural map

$$
H^{1}\left(\tau_{Z_{0}, D_{0} \bar{D}_{0}}^{0}\right) \rightarrow H^{1}\left(\Theta_{Z_{-}, D_{-} \bar{D}_{-} Q_{-}}\right) \oplus H^{1}\left(\Theta_{Z_{+}, D_{+} \bar{D}_{+} Q}\right)
$$

is a surjection. On the other hand, the analogous exact sequence

$$
0 \longrightarrow \tau_{D_{0}}^{0} \longrightarrow q_{*} \Theta_{D_{-} \sqcup D_{-}, \ell_{-} \sqcup \ell_{+}} \longrightarrow i_{*} \Theta_{\ell} \longrightarrow 0
$$

tells us that the natural map

$$
H^{1}\left(\tau_{D_{0}}^{0}\right) \longrightarrow H^{1}\left(\Theta_{D_{-}, \ell_{-}}\right) \oplus H^{1}\left(\Theta_{D_{+}, \ell_{+}}\right)
$$

is an isomorphism; treating $\bar{D}_{0}$ similarly then tells us that

$$
H^{1}\left(\tau_{D_{0} \bar{D}_{0}}^{0}\right)=H^{1}\left(\Theta_{D_{-}, \ell_{-}}\right) \oplus H^{1}\left(\Theta_{\bar{D}_{-}, \bar{\ell}_{-}}\right) \oplus H^{1}\left(\Theta_{D_{+}, \ell_{+}}\right) \oplus H^{1}\left(\Theta_{\bar{D}_{+}, \bar{\ell}_{+}}\right) .
$$

It therefore suffices to show that the natural maps $H^{1}\left(\Theta_{Z_{ \pm}, D_{ \pm} \bar{D}_{ \pm} Q_{ \pm}}\right) \longrightarrow$ $H^{1}\left(\Theta_{D_{ \pm}, \ell_{ \pm}}\right) \oplus H^{1}\left(\Theta_{\bar{D}_{ \pm}, \bar{\ell}_{ \pm}}\right)$are surjective. But as these maps occur in the long exact sequences induced by

$$
0 \longrightarrow \Theta_{Z_{ \pm}, Q_{ \pm}} \otimes \mathcal{I}_{D_{ \pm} \bar{D}_{ \pm}} \longrightarrow \Theta_{Z_{ \pm}, D_{ \pm} \bar{D}_{ \pm} Q_{ \pm}} \longrightarrow \Theta_{D_{ \pm}, \ell_{ \pm}} \oplus \Theta_{\bar{D}_{ \pm}, \bar{\ell}_{ \pm}} \longrightarrow 0
$$


we need merely show that $H^{2}\left(\Theta_{Z_{ \pm}, Q_{ \pm}} \otimes \mathcal{I}_{D_{ \pm} \bar{D}_{ \pm}}\right)=0$.

Now for $Z_{+}$, one has a short exact sequence

$$
0 \longrightarrow \mathcal{V} \otimes \mathcal{I}_{D_{ \pm} \bar{D}_{ \pm}} \longrightarrow \Theta_{Z_{ \pm}, Q_{ \pm}} \otimes \mathcal{I}_{D_{ \pm} \bar{D}_{ \pm}} \longrightarrow \mathcal{O} \longrightarrow 0
$$

where $\mathcal{V}$ denotes the vertical, Q-relative tangent sheaf [17, 3.2]. Since $\pi_{*}^{q} \mathcal{O}=$ $0, q=1,2$, it follows that $H^{j}\left(Z_{+}, \mathcal{O}\right)=H^{j}\left(\mathbf{C P}_{1}, \mathcal{O}\right)$, and thus $H^{2}\left(\Theta_{Z_{+}, Q} \otimes\right.$ $\left.\mathcal{I}_{D_{+} \bar{D}_{+}}\right)=H^{2}\left(\mathcal{V} \otimes \mathcal{I}_{D_{ \pm} \bar{D}_{ \pm}}\right)$. On the other hand [17], $\pi_{*}^{1}\left(\mathcal{V} \otimes \mathcal{I}_{D_{+} \bar{D}_{+}}\right)$vanishes on the complement of two points of $\mathbf{C P}_{1}$, so that $H^{1}\left(\pi_{*}^{1}\left(\mathcal{V} \otimes \mathcal{I}_{D_{+} \bar{D}_{+}}\right)\right)=0$, whereas $\pi_{*}^{2}\left(\mathcal{V} \otimes \mathcal{I}_{D_{+} \bar{D}_{+}}\right)$vanishes outright. The claim therefore follows from the Leray spectral sequence of $\pi: Z_{+} \rightarrow \mathbf{C P}_{1}$.

As for $Z_{-}$, an argument analogous to that of Lemma 2 shows that $H^{2}\left(\Theta_{Z_{-}, Q_{-}} \otimes \mathcal{I}_{D_{-} \bar{D}_{-}}\right) \cong\left[H^{2}\left(\Theta_{\tilde{Z}_{N}, Q} \otimes \mathcal{I}_{D_{N} \bar{D}_{N}}\right)\right]_{\Phi}$. Indeed, for the branched covering map $\alpha$ and blowing down map $\beta$, one can check in a similar way as we have done before that

$\alpha_{*}^{j}\left(\Theta_{\tilde{Z_{N}, Q}} \otimes \mathcal{I}_{\widetilde{D_{N} \bar{D}_{N}}}\right)= \begin{cases}\left(\Theta_{Z_{-}, Q_{-}} \otimes \mathcal{I}_{D_{-} \bar{D}_{-}} \oplus\left[\Theta_{Z_{-}, Q_{-}} \otimes \mathcal{I}_{D_{-} \bar{D}_{-}}\right) \otimes \mathcal{L}\right] & j=0 \\ 0 & j \neq 0,\end{cases}$

while

$$
\beta_{*}^{j}\left(\Theta_{\widetilde{Z_{N}, Q}} \otimes \mathcal{I}_{\bar{D}_{N} \bar{D}_{N}}\right)= \begin{cases}\Theta_{Z_{N}, L_{\Phi}} \otimes \mathcal{I}_{D_{N} \overline{D_{N}}} & j=0 \\ 0 & j \neq 0,\end{cases}
$$

Hence $H^{2}\left(\Theta_{\tilde{Z}_{N}, Q} \otimes \mathcal{I}_{\tilde{D}_{N} \tilde{\bar{D}}_{N}}\right) \cong H^{2}\left(\Theta_{Z_{N}, L_{\Phi}} \otimes \mathcal{I}_{D_{N} \bar{D}_{N}}\right)$. But now the long exact sequence of

$$
0 \longrightarrow \Theta_{Z_{N}, L_{\Phi}} \otimes \mathcal{I}_{D_{N} \bar{D}_{N}} \longrightarrow \Theta_{Z_{N}} \otimes \mathcal{I}_{D_{N} \bar{D}_{N}} \longrightarrow \nu_{L_{\Phi}, Z_{N}} \otimes \mathcal{I}_{D_{N} \bar{D}_{N}} \longrightarrow 0
$$

and the fact that $\nu_{L_{\Phi}, Z_{N}} \otimes \mathcal{I}_{D_{N} \bar{D}_{N}} \cong \mathcal{O}(-1) \oplus \mathcal{O}(-1)$ combine to imply that $H^{2}\left(\Theta_{Z_{N}, L_{\Phi}} \otimes \mathcal{I}_{D_{N} \bar{D}_{N}}\right)=H^{2}\left(\Theta_{Z_{N}} \otimes \mathcal{I}_{D_{N} \bar{D}_{N}}\right)$, so that $H^{2}\left(\Theta_{Z_{-}, Q_{-}} \otimes \mathcal{I}_{D_{-} \bar{D}_{-}}\right) \cong$ $\left[H^{2}\left(\Theta_{\tilde{Z}_{N}, Q} \otimes \mathcal{I}_{D_{N} \bar{D}_{N}}\right)\right]_{\Phi}$, as claimed. Since $H^{2}\left(\Theta_{Z_{N}} \otimes \mathcal{I}_{D_{N} \bar{D}_{N}}\right)=0$ by Theorem 2 , the result follows.

Now, according to Proposition 1, $D_{0}$ has a versal family of deformations over a neighborhood of $0 \in H^{1}\left(D_{-}, \tau_{D_{0}}^{0}\right) \times \mathbf{C}^{k}$ with the property that the fiber over $\left(0, t_{2}\right), t_{2} \in\left(\mathbf{C}^{\times}\right)^{k}$, is biholomorphic to $M$; moreover, the KodairaSpencer map of this family at 0 is compatible with the natural exact sequence

$$
0 \rightarrow H^{1}\left(\tau_{D_{0}}^{0}\right) \rightarrow T_{D_{0}}^{1} \rightarrow H^{0}\left(\tau_{D_{0}}^{1}\right) \rightarrow 0
$$


for which it therefore provides a splitting. By versality, the family $\varpi_{D}$ is therefore induced by a map

$$
T_{f}^{1} \supset U \rightarrow H^{1}\left(\tau_{D_{0}}^{0}\right) \oplus \overline{H^{1}\left(\tau_{D_{0}}^{0}\right)} \oplus \mathbf{C}^{k} \oplus \overline{\mathbf{C}^{k}}
$$

whose derivative at 0 amounts to the natural restriction homomorphism $T_{f}^{1} \rightarrow T_{D_{0} \bar{D}_{0}}^{1}$ and which intertwines the real structure of $T_{f}^{1}$ with the antilinear map on the target which interchanges the obvious pairs of factors. The derivative of the induced map $U \rightarrow H^{1}\left(\tau_{D_{0}}^{0}\right) \oplus \overline{H^{1}\left(\tau_{D_{0}}^{0}\right)}$ is therefore surjective at 0 by Lemma 3; and if we restrict this map to the real slice and then project to the first factor, the resulting map $\left(\Re T_{f}^{1}\right) \cap U \rightarrow H^{1}\left(\tau_{D_{0}}^{0}\right)$ therefore also has surjective derivative at 0 . The inverse image of 0 is thus a $k$-dimensional real submanifold $V$ of $\Re T_{f}^{1}$, and the image of $V$ in

$T_{D_{0} \bar{D}_{0}}^{1}=H^{1}\left(\tau_{D_{0}}^{0}\right) \oplus \overline{H^{1}\left(\tau_{D_{0}}^{0}\right)} \oplus \mathbf{C}^{k} \oplus \overline{\mathbf{C}^{k}}$ is a neighborhood of 0 in a diagonally imbedded $\mathbf{R}^{k} \subset \mathbf{C}^{k} \oplus \overline{\mathbf{C}^{k}}$. Since the generic element of $V$ therefore projects to an element of $\left(\mathbf{C}^{\times}\right)^{k}$, it follows that the restriction of $\varpi_{Z}$ to $V$ is a simultaneous real smoothing of $\left(Z_{0}, D_{0}\right)$ for which the generic hypersurface $D_{t}$ is a copy of $M$. Thus $M$ admits scalar-flat Kähler metrics, and we have proved Theorem 11.

Remark. The above argument actually proves a bit more; namely, any small deformation of $M$ also admits scalar-flat Kähler metrics. This ostensibly stronger statement, however, is actually an immediate formal consequence of the mere statement of Theorem 1 in light of the deformation theory of scalar-flat Kähler surfaces [16] together with Theorem 2.

\section{The Main Theorems}

As a first step toward proving our main results, we now apply the results of the last section to scalar-flat Kähler metrics on some specific surfaces.

Proposition 2 If $\mathbf{C P}_{1} \times \mathbf{C P}_{1}$ is blown up at 13 suitably chosen points, the resulting complex surface admits scalar-flat Kähler metrics. 
Proof. The strategy is to apply Theorem 1 when $N$ is a a two-fold blow-up of $\mathbf{C P}_{1} \times \Sigma$, where $\Sigma$ is a compact complex curve of genus 2 . We therefore begin by constructing such a scalar-flat Kähler metric on this manifold which admits a suitable involution $\Phi$. This will be done by careful use of the hyperbolic ansatz construction of [13].

Let $\Sigma$ be a of genus 2, and let $\phi: \Sigma \rightarrow \Sigma$ be the Weierstraß involution

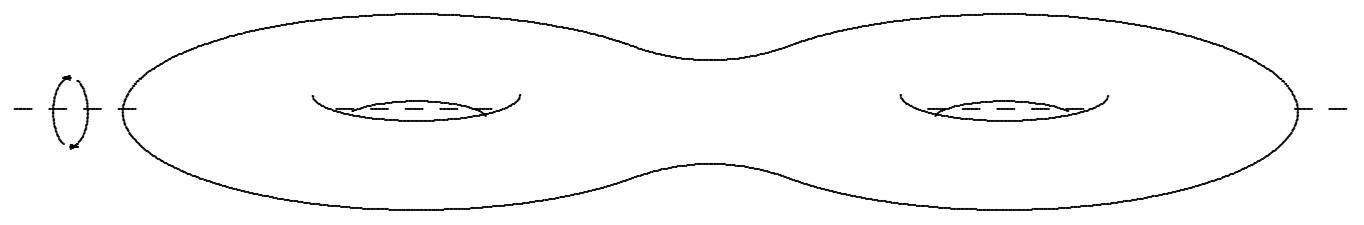

which realizes $\Sigma$ as a 2-sheeted branched cover $\pi: \Sigma \rightarrow \mathbf{C P}_{1}$; let $\hat{q} \in \Sigma$ be one of the 6 fixed points of $\phi$, and set $q=\pi(\hat{q})$. Let $h_{\Sigma}$ be the curvature -1 Hermitian metric on $\Sigma$, and notice that $\phi$ is an isometry of $h_{\Sigma}$.

We now equip the 3 -manifold $X:=\Sigma \times(-1,1)$ with the hyperbolic metric

$$
h=\frac{h_{\Sigma}}{\left(1-t^{2}\right)}+\frac{d t^{2}}{\left(1-t^{2}\right)^{2}},
$$

where $t$ is the standard coordinate on $(-1,1)$. Let $p_{ \pm}=\left(\hat{q}, \pm \frac{1}{2}\right) \in X$, let $G_{ \pm}$ be the hyperbolic Green's functions of $p_{ \pm} \in X$, and set $V=1+G_{+}+G_{-}$. We then let $P$ be the principal $S^{1}$-bundle on $X-\left\{p_{ \pm}\right\}$with connection 1-form $\theta$ such that

$$
\star d V=d \theta
$$

and such that the restriction of $(P, \theta)$ to the hypersurface $t=0$ is the trivial bundle-with-connection $\Sigma \times S^{1}$. We then endow $P$ with the Riemannian metric

$$
g=\left(1-t^{2}\right)\left[V h+V^{-1} \theta^{2}\right] .
$$

The metric space completion of $(P, g)$ is then a smooth compact Riemannian 4-manifold $\left(N, g_{N}\right)$ of scalar-curvature zero, and admits a complex structure $J_{N}$ with respect to which $g_{N}$ is Kähler. Moreover [13], the complex surface $\left(N, J_{N}\right)$ is biholomorphic to $\mathbf{C P}_{1} \times \Sigma$ blown up at two points in the fiber over $q \in \Sigma$. 
Consider the map $\psi: \Sigma \times(-1,1) \rightarrow \Sigma \times(-1,1)$ given by $(\zeta, t) \mapsto$ $(\phi(\zeta),-t)$. As $\psi^{*} V=V$ and $\psi$ is an orientation-reversing isometry of $X$, it follows that $\psi^{*} P \cong \bar{P}$ as a principal bundle-with-connection, where $\bar{P}$ denotes $P$ equipped with the inverse $S^{1}$-action. There is therefore a unique isometry $\Phi$ of $P$ which covers $\psi$ and restricts to the hypersurface $t=0$ as $\phi \times c: \Sigma \times S^{1} \rightarrow \Sigma \times S^{1}$, where $c: S^{1} \rightarrow S^{1}$ is the reflection $e^{i \vartheta} \rightarrow e^{-i \vartheta}$. This extends to the Riemannian completion $N$ as an involution of the desired type. Indeed, it is not hard to see that $\Phi$ is induced by the involution $r \times \phi$ of $\mathbf{C P}_{1} \times \Sigma$, where $r$ is $180^{\circ}$ rotation of $\mathbf{C P}_{1}=S^{2}$ about an axis,

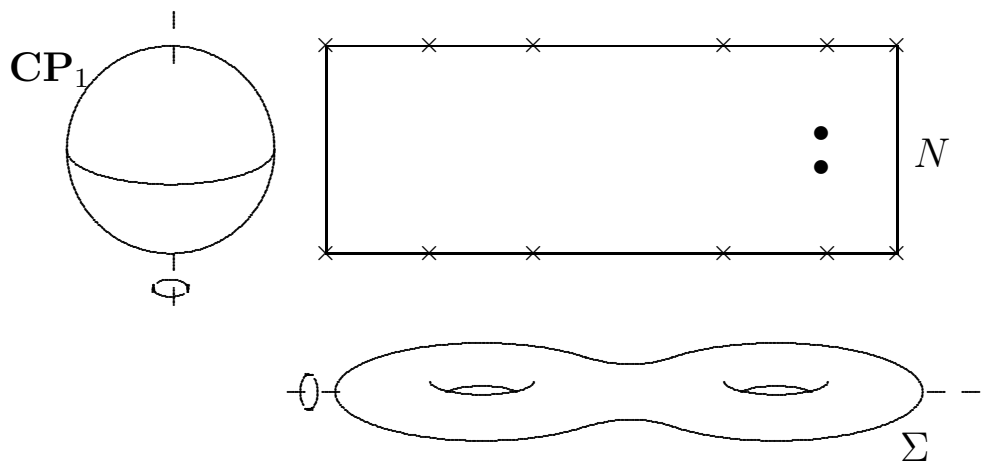

which interchanges the two blown-up points, which are antipodal on the equator of an invariant $S^{2}$.

One may now apply Theorem 1, but it remains for us to understand the structure of the resulting $\left(M, J_{M}\right)$. In order to do this, first blow up $N$ at the 12 fixed points of $\Phi$, and notice that we have the following arrangement of curves in the blow-up $\tilde{N}$ :

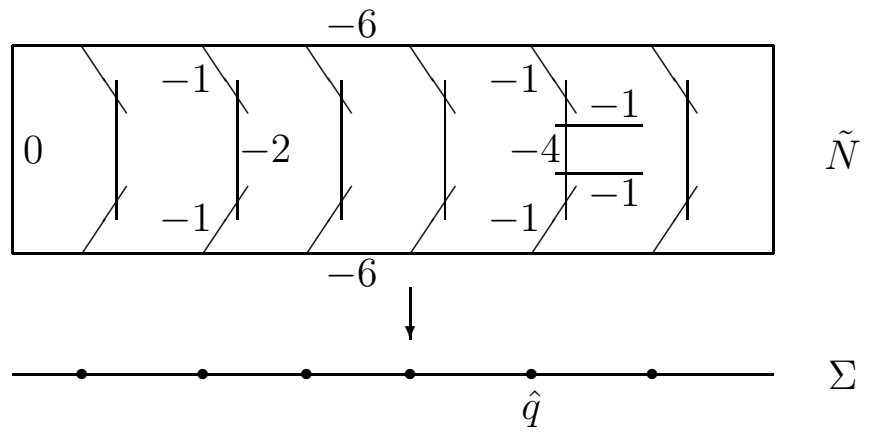


Now $\tilde{N}$ is a 2-fold branched cover of $M$, with ramification locus equal to the twelve $(-1)$-curves introduced by the blowing up the fixed points. Descending to $M$ will thus double the self-intersection of these branch curves, while halving the self-intersection of any curve on which $\Phi$ acts non-trivially. The corresponding picture of $M$ is therefore as follows:

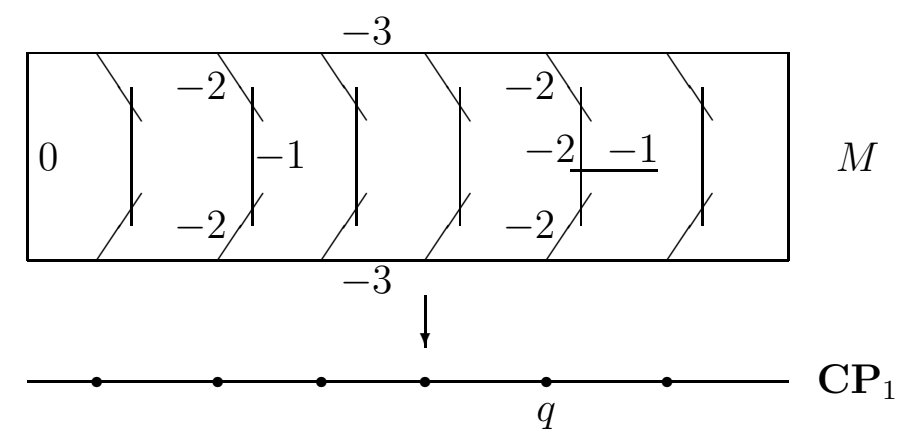

Contracting 13 judiciously chosen exceptional curves,

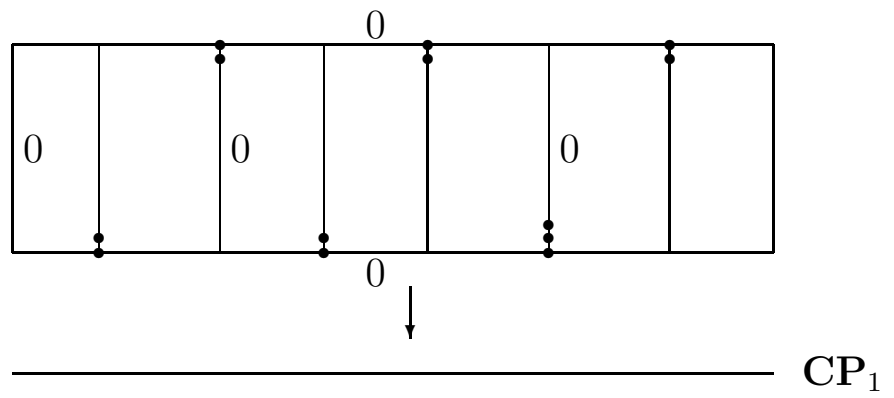

we get $\mathbf{C P}_{1} \times \mathbf{C P}_{1}$ as our minimal model. Invoking Theorem [1, we thus conclude that the above iterated blow-up of $\mathbf{C P}_{1} \times \mathbf{C P}_{1}$ admits scalar-flat Kähler metrics. Moreover, it also follows from our smoothing argument that any sufficiently small deformation 


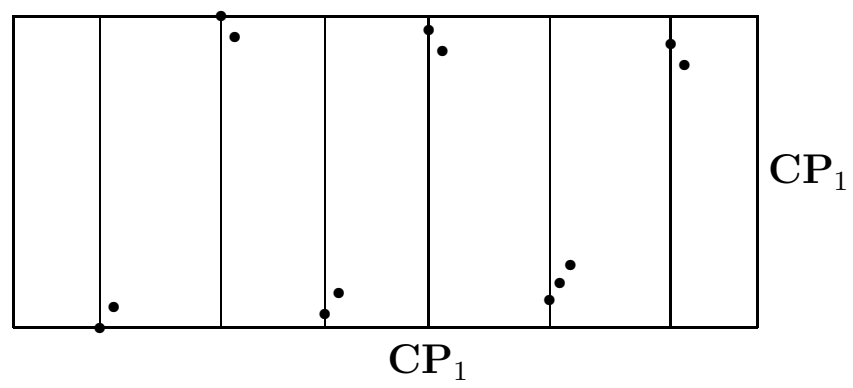

of $M$ also admits scalar-flat Kähler metrics.

Corollary 1 If $\mathbf{C P}_{2}$ is blown up at 14 suitably chosen points, the resulting complex surface admits scalar-flat Kähler metrics.

Proof. The blow-up of $\mathbf{C P}_{2}$ at two distinct points is biholomorphic to the blow-up of $\mathbf{C P}_{1} \times \mathbf{C P}_{1}$ at one point. Now use Proposition 2 .

Proposition 3 Let $E \approx T^{2}$ be any elliptic curve. If $E \times \mathbf{C P}_{1}$ is blown up at 6 suitably chosen points, the resulting complex surface admits scalar-flat Kähler metrics.

Proof. Our strategy is similar to that used in the genus 0 case, but we will now have to exercise great care in order to compensate for the non-trivial Jacobi variety of $E$.

We begin by choosing 4 distinct points $q_{1}, q_{2}, r_{1}, r_{2} \in E$ such that $q_{1}+q_{2}=$ $r_{1}+r_{2}$ as divisors, and a holomorphic line bundle $L \rightarrow E$ which is a squareroot of the divisor $q_{1}+q_{2}=r_{1}+r_{2}$. (For example, if $E$ is $\mathbf{C} / \Lambda$, where lattice $\Lambda$ is generated by 1 and $\tau$, we may take $q_{1}, q_{2}, r_{1}, r_{2}$ to respectively be the equivalence classes of $0,(1+\tau) / 2,1 / 2$, and $\tau / 2$, whereas $L$ may be taken to be the divisor of the point represented by $(1+\tau) / 4$.) Thus $L^{\otimes 2}$ comes equipped with holomorphic sections $u_{1}$ and $u_{2}$ whose zero sets are respectively $\left\{q_{1}, q_{2}\right\}$ and $\left\{r_{1}, r_{2}\right\}$, and all these zeroes are simple. We now let 
$\Sigma$ be the 2-fold branched cover of $E$ with branch locus $\left\{q_{1}, q_{2}\right\}$ associated to the bundle $L$; explicitly,

$$
\Sigma=\left\{\zeta \in L \mid \zeta \otimes \zeta=u_{1} \pi_{L}(\zeta)\right\}
$$

where $\pi_{L}: L \rightarrow E$ is the canonical projection. Let $\phi: \Sigma \rightarrow \Sigma$ be the involution with 2 fixed points induced by multiplication by -1 in $L$, and let $\pi$ : $\Sigma \rightarrow E$ denote the canonical projection induced by $\pi_{L}$. Let $\pi^{-1}\left(\left\{q_{1}, q_{2}\right\}\right)=$ $\left\{\hat{q}_{1}, \hat{q}_{2}\right\}$, and let $\pi^{-1}\left(\left\{r_{1}, r_{2}\right\}\right)=\left\{\hat{r}_{1}, \hat{r}_{2}, \hat{r}_{3}, \hat{r}_{4}\right\}$. Notice that $\pi^{*} L$ is the divisor line bundle of $\hat{q}_{1}+\hat{q}_{2}$, whereas its square $\pi^{*} L^{\otimes 2}$ is the divisor of $\hat{r}_{1}+\hat{r}_{2}+\hat{r}_{3}+\hat{r}_{4}$.

As before, we equip the 3 -manifold $X:=\Sigma \times(-1,1)$ with the hyperbolic metric

$$
h=\frac{h_{\Sigma}}{\left(1-t^{2}\right)}+\frac{d t^{2}}{\left(1-t^{2}\right)^{2}},
$$

but this time we set $p_{j}=\left(\hat{q}_{j}, 0\right), j=1, \ldots, 4$. Let $G_{j}$ be the hyperbolic Green's functions of $p_{j} \in X$, and set $V=1+\sum_{j=1}^{4} G_{j}$. On $(\Sigma \times\{0\})-\left\{p_{j}\right\} \cong$ $\Sigma-\left\{\hat{q}_{j}\right\}$, let $P_{0}$ be the flat $S^{1}$ bundle with $\mathbf{Z}_{2}$ monodromy corresponding to the branched cover with branch-points $\hat{r}_{1}, \ldots, \hat{r}_{4}$ associated with the line bundle $\pi^{*} L$. Then [13] there exists a principal $S^{1}$-bundle $P \rightarrow X-\left\{p_{j}\right\}$ with connection 1-form $\theta$ such that

$$
\star d V=d \theta
$$

and such that the restriction of $(P, \theta)$ to the hypersurface $t=0$ is the flat bundle $P_{0}$. Indeed, the Chern-Weil theorem guarantees that we can find a connection with curvature $\star d V$ because $d \star d V=0$ on $X-\left\{p_{j}\right\}$ and $\left[\frac{1}{2 \pi}\right] \in H^{2}\left(X-\left\{p_{j}\right\}, \mathbf{Z}\right)$. Because $V$ is symmetric in $t$, a connection with curvature $\star d V$ is automatically flat on $(\Sigma \times\{0\})-\left\{p_{j}\right\}$, and its holonomy around $p_{j}$ is automatically $e^{i \int_{D} \star d V}=e^{\frac{i}{2} \int_{S} \star d V}=e^{\frac{i}{2} \int_{B} d \star d V}=e^{-i \pi}=-1$, where $D$ is a disk in $t \geq 0$ that bounds a loop around $p_{j}$ in $\Sigma \times\{0\}, S$ is the sphere made up of $D$ and its reflection in $t$, and $B$ is the ball about $p_{j} \in X$ with boundary $S$. Twisting by the pull-back of a flat connection on $\Sigma$ now allows us to modify the the restriction of $(P, \theta)$ to $\Sigma \times\{0\}$ so as to obtain any given flat connection with holonomy -1 round the points $p_{j}$; and $P_{0}$ fits the bill. 
As before, we now endow $P$ with the Riemannian metric

$$
g=\left(1-t^{2}\right)\left[V h+V^{-1} \theta^{2}\right]
$$

The metric space completion of $(P, g)$ is then a smooth compact Riemannian 4-manifold $\left(N, g_{N}\right)$ of scalar-curvature zero, and admits a complex structure $J_{N}$ with respect to which $g_{N}$ is Kähler. There is a smooth holomorphic curve $\hat{\Sigma} \subset N$ which is a copy of the two-fold cover of $\Sigma$ with branch points $\hat{r}_{1}, \ldots, \hat{r}_{4}$ associated with $\pi^{*} L$, obtained by taking closure of the $\mathbf{Z}_{2}$ bundle from which $P_{0}$ was constructed. This allows one to observe [13] that $\left(N, J_{N}\right)$ is obtained from $\mathbf{P}\left(\pi^{*} L \oplus \mathcal{O}\right) \rightarrow \Sigma$ by blowing up the points $\hat{r}_{1}, \ldots, \hat{r}_{4}$ on the zero section of $\pi^{*} L \subset \mathbf{P}\left(\pi^{*} L \oplus \mathcal{O}\right)$; the key point is that $\hat{\Sigma} \subset N$ corresponds to the proper transform of the curve $\zeta^{\otimes 2}=u_{3}$, where $u_{3} \in \Gamma\left(\Sigma, \mathcal{O}\left(\pi^{*} L^{\otimes 2}\right)\right)$ is the standard section with simple zeroes at $\hat{r}_{1}, \ldots, \hat{r}_{4}$.

Consider the involution $\iota: \pi^{*} L \rightarrow \pi^{*} L$ which maps $\left(\pi^{*} L\right)_{z}$ to $\left(\pi^{*} L\right)_{\phi(z)}$ by multiplication by -1 in $L_{\pi(z)}=L_{\pi(\phi(z))}$. This involution extends $\mathbf{P}\left(\pi^{*} L \oplus\right.$ $\mathcal{O})$, and so lifts to an involution $\Phi: N \rightarrow N$ with exactly 4 fixed points, corresponding to 0 and $\infty$ in the fibers over $\hat{q}_{1}$ and $\hat{q}_{2}$. Since $\pi^{*} L$ is the divisor line bundle of $\hat{q}_{1}+\hat{q}_{2}$, there is a section $u$ of $\pi^{*} L$ with simple zeroes at $\hat{q}_{1}$ and $\hat{q}_{2}$, and by averaging we may arrange that $\iota(u(z))=-u(\phi(z))$ for all $z \in \Sigma$; the proper transform $C_{u} \subset N$ of the image of $u$ is then $\Phi$-invariant.

Now the involution $\Phi$ just corresponds to the unique connection-preserving involution of $P$ which covers $\phi \times 1:[\Sigma \times(-1,1)] \rightarrow[\Sigma \times(-1,1)]$ and extends $\iota: \hat{\Sigma} \rightarrow \hat{\Sigma}$. It therefore preserves $g_{N}$, and we may apply Theorem 11. The remaining task is thus to analyze the structure of the surface $\left(M, J_{M}\right)$.

Since $\tilde{N}$ is obtained from $N$ by blowing up the 4 fixed points of $\Phi$, it contains the following arrangement of curves:

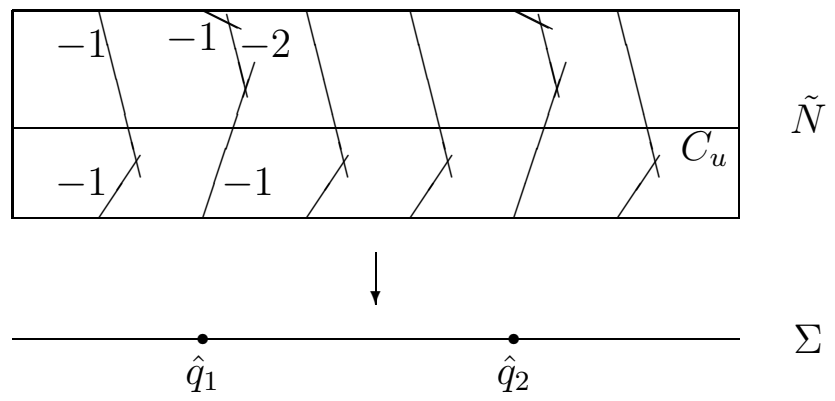


The corresponding picture of $M$ is therefore:

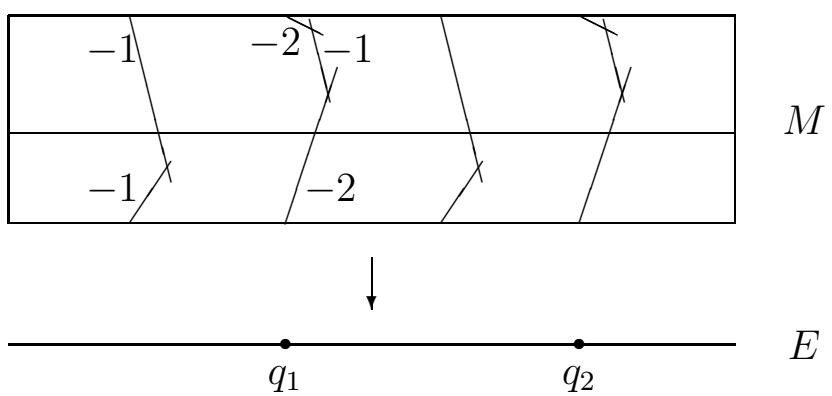

Contracting 6 exceptional curves in the right order,

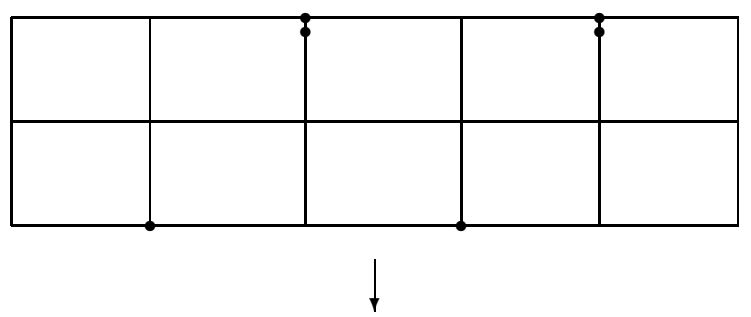

we get $E \times \mathbf{C P}_{1}$ as our minimal model. Theorem 1 thus tells us that the above iterated blow-up of $E \times \mathbf{C P}_{1}$ admits scalar-flat Kähler metrics, as do its sufficiently small deformations.

Remark. Notice that the scalar-flat Kähler surface we have just constructed admits a holomorphic $\mathbf{C}^{\times}$-action; namely, the $\mathbf{C}^{\times}$-action on $\mathbf{C P}_{1} \times E$ induced by the "earth-rotation" of $\mathbf{C P}_{1}$ lifts to $M$, which is obtained from $\mathbf{C P}_{1} \times E$ by iteratively blowing up fixed points of the action. Also notice, however, that the induced action is not semi-free, since the isotropy of any generic point on either of the second-level blow-up curves is $\{ \pm 1\} \subset \mathbf{C}^{\times}$. A theorem of Lichnerowicz [18] now implies that $S^{1} \subset \mathbf{C}^{\times}$acts isometrically on this scalarflat Kähler surface. By combining elements of the proofs of Propositions 2 and 3, one can similarly construct scalar-flat Kähler metrics with non-semifree $S^{1}$-action on 14-point blow-ups of $\mathbf{C P}_{1} \times \mathbf{C P}_{1}$. 
One might, in principle, use the Toda lattice equation [12 to construct the above metrics explicitly. However, Theorem 2 of [14] insists that these solutions definitely cannot arise from the hyperbolic ansatz case of that construction. This is consistent with the fact that the orbits with exceptional isotropy give rise to very peculiar orbifold singularities of the associated 3dimensional Einstein-Weyl geometry.

Any of these metrics is a counter-example to the assertion [16, Proposition 3.1] that a blown-up ruled surface of genus $<2$ cannot admit both a $\mathbf{C}^{\times}$action and a Kähler class of total scalar curvature 0 . While the argument offered there does indeed work if one insists that the $\mathbf{C}^{\times}$-action be semi-free, the recipe given for a section of the anti-canonical bundle simply breaks down if, as in the present case, an isolated fixed point is blown up at some stage in the iterated blow-up process.

Theorem 3 Let $(M, J)$ be a ruled surface-i.e. suppose that $M$ is a compact complex 2-manifold for which there exists a holomorphic map $M \rightarrow \Sigma$ with generic fiber $\mathbf{C P}_{1}$ and range a Riemann surface $\Sigma$. Then $(M, J)$ has blow-ups $(\tilde{M}, \tilde{J})$ which admit scalar-flat Kähler metrics.

Proof. Any ruled surface $M$ is bimeromorphic [泪 to some product surface $\Sigma \times \mathbf{C P}_{1}$. If $M_{1}$ is any blow-up of $\Sigma \times \mathbf{C P}_{1}$, it then follows that there is a blow-up $M_{2}$ of $M_{1}$ which is also a blow-up of $M$ :

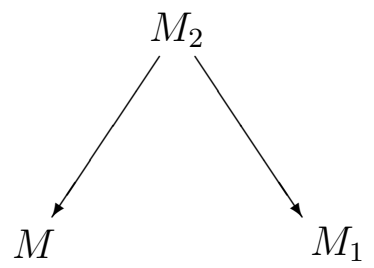

But [11, Theorem 4.6] and Theorem 2 tell us that any 1-point blow-up of a non-minimal scalar-flat Kähler surface also admits Kähler metrics; induction then says the same is also true of iterated blow-ups. It therefore suffices to find just one blow-up $M_{1}$ of each $\Sigma \times \mathbf{C P}_{1}$ which admits a scalar-flat Kähler metric. If the genus of $\Sigma$ is at least 2, the hyperbolic ansatz [13] then 
explicitly constructs such metrics on a 2-point blow-up $M_{1}$ of $\Sigma \times \mathbf{C P}_{1}$. For genera 0 and 1 , on the other hand, the necessary surfaces $M_{1}$ are constructed in Propositions 2 and 3 .

Theorem A Let $(M, J)$ be a compact complex 2-manifold which admits a Kähler metric for which the integral of the scalar curvature is non-negative. Then precisely one of the following holds:

- $(M, J)$ admits a Ricci-flat Kähler metric; or

- any blow-up of $(M, J)$ has blow-ups $(\tilde{M}, \tilde{J})$ which admit scalar-flat Kähler metrics. Moreover, any blow-up of such an $(\tilde{M}, \tilde{J})$ admits scalar-flat Kähler metrics, too.

Proof. By a vanishing theorem of Yau [29], any compact Kähler surface of non-negative total scalar curvature is either ruled or has $c_{1}^{\mathbf{R}}=0$. In the latter case, Yau's solution of the Calabi conjecture [30] guarantees the existence of a Ricci-flat metric on $M$. If, on the other hand, $M$ is ruled, so is any blow-up $\hat{M}$ of $M$; and Theorem 3 tells us that some blow-up $\tilde{M}$ of $\hat{M}$ therefore admits a scalar-flat Kähler metric. Finally, any blow-up of a non-minimal scalar-flat Kähler surface also admits scalar-flat Kähler metrics, by [11, Theorem 4.6] and Theorem 2, and this may be appied to $\tilde{M}$ to prove the last clause.

Theorem B Let $(M, J)$ be a compact complex 2-manifold which admits a Kähler metric for which the integral of the scalar curvature is positive. Then any blow-up of $(M, J)$ has blow-ups $(\tilde{M}, \tilde{J})$ which admit Kähler metrics of constant positive scalar curvature. Moreover, any blow-up of such an $(\tilde{M}, \tilde{J})$ also admits such metrics.

Proof. The hypothesis says that $c_{1} \cdot[\omega]>0$ for some Kähler class $[\omega]$, so that $c_{1}^{\mathbf{R}} \neq 0$ and $M$ certainly cannot admit a Ricci-flat metric. On the other hand, a straightforward inverse-function-theorem argument 15] shows that if a compact complex manifold admits a non-Ricci-flat scalar-flat Kähler metric, it also admits Kähler metrics of constant positive scalar curvature. The result therefore follows from Theorem $\mathrm{A}$. 


\section{Appendix: The Vanishing Theorem}

In this appendix, we demonstrate that Kodaira-Spencer theory is unobstructed for the twistor spaces of all non-minimal scalar-flat Kähler surfaces. The proof is a direct extension of that presented in [16], but allows for the possibility of non-semi-free $\mathbf{C}^{\times}$-actions.

Theorem 2 Suppose that $N$ is a non-minimal compact complex surface with scalar-flat Kähler metric $g_{N}$. Then its twistor space satisfies $H^{2}\left(Z_{N}, \Theta\right)=$ $H^{2}\left(Z_{N}, \Theta \otimes \kappa^{-1 / 2}\right)=H^{2}\left(Z_{N}, \Theta_{Z, D \bar{D}}\right)=0$, where $D$ and $\bar{D}$ are canonical divisors associated with $J_{N}$ and $-J_{N}$.

Proof. The natural homomorphisms

$$
\begin{aligned}
H^{2}\left(Z_{N}, \Theta \otimes \kappa^{-1 / 2}\right) & \rightarrow H^{2}\left(Z_{N}, \Theta_{Z, D \bar{D}}\right), \\
H^{2}\left(Z_{N}, \Theta_{Z, D \bar{D}}\right) & \rightarrow H^{2}\left(Z_{N}, \Theta\right)
\end{aligned}
$$

are surjective because $H^{2}\left(N, \Theta_{N}\right)=H^{2}\left(N, \mathcal{O}\left(\kappa_{N}^{-1}\right)\right)=0$ for any ruled surface $N$. On the other hand, careful inspection of the Penrose transform shows [16, Theorem 2.7] that $H^{2}\left(Z_{N}, \Theta \otimes \kappa^{-1 / 2}\right)$ is canonically identified with the kernel of

$$
\left.d \mathcal{F}\right|_{[\omega]}: H^{0}\left(N, \Theta_{N}\right) \rightarrow A^{*},
$$

where $\mathcal{F}$ is the Futaki invariant, $[\omega]$ is the Kähler class, and $A \subset H^{1,1}(N)=$ $H^{2}(N)$ is the hyperplane

$$
\left\{\alpha \in H^{1,1}(N) \mid c_{1} \cup \alpha=0\right\} .
$$

Our goal here will thus be to show that (5.4) is injective.

Because [18 the automorphism group of $N$ has a compact real form given by the isometry goup of $g_{N}, H^{0}\left(N, \Theta_{N}\right)$ is spanned by vector fields $\Xi$ which generate $\mathbf{C}^{\times}$actions which are free on an open dense, and such that the $S^{1}$-action generated by $\xi=\Im \Xi$ is isometric with respect to $g_{N}$, while $\Re \Xi$ is globally a gradient vector field because the contraction of $\Xi$ with any holomorphic 1-form vanishes identically. In fact, $H^{0}\left(N, \Theta_{N}\right)$ is 
at most 1-dimensional; for if $\Xi_{1}$ and $\Xi_{2}$ are two such fields, we must have $\Xi_{1} \wedge \Xi_{2} \equiv 0$ because [16, 29] the existence of a scalar-flat Kähler metric forces $H^{0}\left(N, \kappa^{-1}\right)=0$. This implies that the generic orbit of $\Xi_{1}$ has closure $F \cong \mathbf{C P}_{1}$ which is also the closure of a generic orbit of $\Xi_{2}$. If $\Xi_{1}$ does not have the same zeroes on this $\mathbf{C P}_{1}$ as $\Xi_{2}$, the isometry goup contains an $S U(2)$ which acts transitively on this 2-sphere, and the orbits of this $S U(2)$ are all either holomorphically embedded $\mathbf{C P}_{1}$ 's or points; but the latter type of orbit is impossible, because an $S^{2}=\mathbf{C P}_{1}$ orbit near a fixed point would be contained in the domain of a holomorphic chart, contradicting the fact that every holomorphic function on $\mathbf{C P}_{1}$ is constant. Thus every orbit of this $S U(2)$ would be a $\mathbf{C P}_{1}$, and we would conclude that $N$ would be a minimal ruled surface - contradicting our hypotheses. Thus $\Xi_{1}$ and $\Xi_{2}$ must have the same zeroes on the generic orbit-curve $F$, and hence, since both generate generically free $\mathbf{C}^{\times}$-actions, $\Xi_{1}= \pm \Xi_{2}$. This shows that $h^{0}\left(N, \Theta_{N}\right) \leq 1$, as claimed.

As there is nothing to show if $H^{0}\left(N, \Theta_{N}\right)=0$, we may therefore assume henceforth that $H^{0}\left(N, \Theta_{N}\right) \cong \mathbf{C}$ and is spanned by a holomorphic vector field $\Xi$ whose imaginary part $\xi$ is a periodic Killing field, with generic minimal period $2 \pi$. By averaging, we can represent any other Kähler class by a metric $g$ which is $S^{1}$-invariant. With respect to such a metric $g$, whose Kähler form we shall call $\omega$, let $t$ be the Hamiltonian function of $\xi$, conventionally normalized so as to have range of the form $[-a, a]$, and let $\Sigma$ be the stable quotient $N / / \mathbf{C}^{\times}$. If $t=a$ and/or $t=-a$ are isolated fixed points, blow them up to obtain a new complex surface $\hat{N}$; otherwise, set $N=\hat{N}$. We then have a map $\Pi: \hat{N} \rightarrow \Sigma \times[-a, a]$ with $S^{1}$-orbits as fibers. If $p_{1}, \ldots, p_{m}$ are the images in $\Sigma \times(-a, a)$ of the isolated fixed points, and if $X=[\Sigma \times(-a, a)]-\left\{p_{j}\right\}$, then on the open dense set $Y=\Pi^{-1}(X) \subset \hat{N}$ we may express the given Kähler metric $g$ in the form

$$
g=w \hat{g}(t)+w d t^{\otimes 2}+w^{-1} \theta^{\otimes 2}
$$

for some positive functions $w>0$ on $X$ and a family orbifold metrics $\hat{g}(t)$ on $\Sigma$. Here $\theta$ is the unique 1 -form on $Y$ whose kernel is orthogonal to $\xi$ and such that $\theta(\xi)=1$. The orbifold points in $X$ of the $\hat{g}(t)$ exactly correspond 
those $S^{1}$-orbits in $M$ which are non-trivial, but which have period $2 \pi / n$ for some $n$. Since these also coincide with points at which the $\mathbf{C}^{\times}$-action has exceptional isotropy, the orbifold points of $X$ exactly consist of vertical line segments in $\Sigma \times(-a, a)$ which join two of the $p_{j}$. If we let $\jmath$ range over the set of exceptional curves contained in fibers of $\hat{N} \rightarrow \Sigma$, each such curve is the closure of a $\mathbf{C}^{\times}$-orbit, and so we may define integers $m_{\jmath} \geq 1$ as the order of the associated isotropy group, and real numbers $t_{\jmath}^{-}<t_{\jmath}^{+}$, defined to be the minimum and maximum values, respectively, of the Hamiltonian $t$ on the associated exceptional rational curve $E_{\jmath}$. Also define

$$
m_{\jmath}(t)= \begin{cases}m_{\jmath} & t_{\jmath}^{-}<t<t_{\jmath}^{+} \\ 1 & \text { otherwise }\end{cases}
$$

Finally, let $t_{j}$ be the $t$ coordinate of $p_{j}$.

Because $g, w$ and $d t$ are geometrically defined, $\hat{g}(t)$ is an invariantly defined, $t$-dependent orbifold Kähler metric on $\Sigma$ for all $t \notin\left\{t_{j}\right\}$; and when when $t \in\left\{t_{j}\right\}$, it is and defined everywhere aside from a fine number of punctures. Let $\hat{\omega}(t)$ be the Kähler form of $\hat{g}(t)$. If $C^{-}$and $C^{+}$denote the "repulsive" and "attractive" curves $t= \pm a$ in $\hat{N}$, we then have [16]

$$
\begin{aligned}
\left.\hat{\omega}\right|_{t= \pm a} & =0 \\
\left.\frac{d}{d t} \hat{\omega}\right|_{t=-a} & =\left.2 \omega\right|_{C^{-}} \\
\left.\frac{d}{d t} \hat{\omega}\right|_{t=a} & =-\left.2 \omega\right|_{C^{+}},
\end{aligned}
$$

while the density of scalar curvature may be written globally on $Y \subset M$ as

$$
s d \mu=\left[-2 \hat{\rho}+\frac{d^{2}}{d t^{2}} \hat{\omega}\right] \wedge d t \wedge \theta .
$$

Here the Ricci form $\hat{\rho}(t)$ of $\hat{g}(t)$ satisfies

$$
\frac{1}{2 \pi} \int_{\Sigma} \hat{\rho}(t)=\chi(\Sigma)-\sum_{\jmath}\left(1-\frac{1}{m_{\jmath}(t)}\right)
$$


for $t \notin\left\{t_{j}\right\}$ by the Gauss-Bonnet Theorem for orbifolds [24]. Thus

$$
\begin{aligned}
\int_{N} t s d \mu= & \int_{Y} t s d \mu \\
= & \int_{Y} t\left[-2 \hat{\rho}+\frac{d^{2}}{d t^{2}} \hat{\omega}\right] \wedge d t \wedge \theta \\
= & 2 \pi \int_{\Sigma} \int_{-a}^{a} t \frac{d^{2}}{d t^{2}} \hat{\omega} d t-4 \pi \int_{-a}^{a} t\left[\int_{\Sigma} \hat{\rho}\right] d t \\
= & 2 \pi \int_{\Sigma}\left(\left[t \frac{d}{d t} \hat{\omega}\right]_{-a}^{a}-\int_{-a}^{a} \frac{d \hat{\omega}}{d t} d t\right) \\
& \quad-4 \pi \int_{-a}^{a} 2 \pi\left[\chi(\Sigma)-\sum_{\jmath}\left(1-\frac{1}{m_{\jmath}(t)}\right)\right] t d t \\
= & 2 \pi \int_{\Sigma}\left[t \frac{d}{d t} \hat{\omega}-\hat{\omega}\right]_{-a}^{a}-8 \pi^{2} \sum_{\jmath} \frac{1}{m_{\jmath}} \int_{t_{\jmath}^{-}}^{t^{+}} t d t \\
= & 2 \pi a\left[\int_{C^{-}} 2 \omega-\int_{C^{+}} 2 \omega\right]-4 \pi^{2} \sum_{\jmath} \frac{1}{m_{\jmath}}\left[\left(t_{\jmath}^{+}\right)^{2}-\left(t_{\jmath}^{-}\right)^{2}\right] \\
= & {\left[\int_{C^{-}} \omega-\int_{C^{+}} \omega\right] \int_{F} \omega+2 \pi \sum_{\jmath}\left[\left(a-t_{\jmath}^{+}\right)-\left(t_{\jmath}^{-}-(-a)\right)\right] \int_{E_{\jmath}} \omega } \\
= & {\left[\int_{C^{-}} \omega-\int_{C^{+}} \omega\right] \int_{F} \omega+\sum_{\jmath}\left[\sum_{\imath \gg \jmath} m_{\imath} \int_{E_{\imath}} \omega-\sum_{\imath l_{\jmath}} m_{\imath} \int_{E_{\imath}} \omega\right] \int_{E_{\jmath}} \omega } \\
= & {\left[\int_{C^{-}} \omega-\int_{C^{+}} \omega\right] \int_{F} \omega+\sum_{\jmath ; l_{\jmath}}\left(m_{\jmath}-m_{\imath}\right)\left[\int_{E_{\imath}} \omega\right] \int_{E_{\jmath}} \omega . }
\end{aligned}
$$

Here $F$ is any smooth fiber of $\hat{N} \rightarrow \Sigma$, and the partial ordering 1 on $\{\jmath\}$ means that, with respect to the flow of $\Re \Xi$, the first exceptional curve precedes the second in some singular fiber of $\hat{N} \rightarrow \Sigma$.

On the other hand, the total scalar curvature of such a metric $g$ is

$$
\begin{aligned}
\int_{N} s d \mu & =\int_{Y} s d \mu \\
& =\int_{Y}\left[-2 \hat{\rho}+\frac{d^{2}}{d t^{2}} \hat{\omega}\right] \wedge d t \wedge \theta \\
& =2 \pi \int_{\Sigma} \int_{-a}^{a} \frac{d^{2}}{d t^{2}} \hat{\omega} d t-4 \pi \int_{-a}^{a} \int_{\Sigma} \hat{\rho} d t
\end{aligned}
$$




$$
\begin{aligned}
& =2 \pi \int_{\Sigma}\left[\frac{d}{d t} \hat{\omega}\right]_{-a}^{a}-4 \pi \int_{-a}^{a} 2 \pi\left[\chi(\Sigma)-\sum_{\jmath}\left(1-\frac{1}{m_{\jmath}(t)}\right)\right] d t \\
& =-2 \pi\left[\int_{C^{-}} 2 \omega+\int_{C^{+}} 2 \omega\right]-16 \pi^{2} a \chi(\Sigma)+8 \pi^{2} \sum_{\jmath}\left(1-\frac{1}{m_{\jmath}}\right)\left(t_{\jmath}^{+}-t_{\jmath}^{-}\right) \\
& =-4 \pi\left[\int_{C^{-}} \omega+\int_{C^{+}} \omega+2(1-\mathbf{g}) \int_{F} \omega+\sum_{\jmath}\left(1-m_{\jmath}\right) \int_{E_{\jmath}} \omega\right],
\end{aligned}
$$

where $\mathbf{g}$ denotes the genus of $\Sigma$. When the above vanishes, we then have

$$
\begin{aligned}
\mathcal{F}(\Xi,[\omega]) & :=-\frac{1}{2} \int_{N} t\left(s-s_{0}\right) d \mu=-\frac{1}{2} \int_{N} t s d \mu \\
& =\frac{1}{2}\left[\int_{C^{+}} \omega-\int_{C^{-}} \omega\right] \int_{F} \omega+\frac{1}{2} \sum_{\jmath ; \mathbf{l} \jmath}\left(m_{\imath}-m_{\jmath}\right)\left[\int_{E_{\imath}} \omega\right] \int_{E_{\jmath}} \omega .
\end{aligned}
$$

(Here $s_{0}=\int s d \mu / \int d \mu$ denotes the average value of the scalar curvature.) If the $\mathbf{C}^{\times}$-action generated by $\Xi$ is semi-free, all the $E_{\jmath}$ terms drop out, and the formula simplifies to yield that of [16, Theorem 3.2].

Let $\mathcal{F}:=\mathcal{F}(\Xi, \cdot)$ and let $\mathcal{S}=\frac{-1}{4 \pi} \int s d u=-c_{1} \cdot[\omega]$, thought of as functions on the Kähler cone $\subset H^{1,1}(N, \mathbf{R})$. Letting $\Omega=[\omega]$ denote the Kähler class, we therefore have

$$
\mathcal{S}(\Omega)=\Omega\left(C^{-}+C^{+}+2(1-\mathbf{g}) F+\sum\left(1-m_{\jmath}\right) E_{\jmath}\right)
$$

and

$$
2 \mathcal{F}(\Omega)=\Omega(F) \Omega\left(C^{+}-C^{-}\right)+\sum_{\jmath ; \mathbf{l}_{\jmath}}\left(m_{\imath}-m_{\jmath}\right) \Omega\left(E_{\imath}\right) \Omega\left(E_{\jmath}\right)
$$

provided that $\mathcal{S}(\Omega)=0$. Thus, when $\Omega \in H^{1,1}(N, \mathbf{R})$ is the Kähler class of a scalar-flat Kähler metric $g_{N}$,

$$
d \mathcal{S}=C^{-}+C^{+}+2(1-\mathbf{g}) F+\sum\left(1-m_{\jmath}\right) E_{\jmath}
$$

and

$$
\left.2 d \mathcal{F}\right|_{\Omega} \equiv \Upsilon \bmod d \mathcal{S}
$$

where

$$
\Upsilon:=\Omega(F)\left[C^{+}-C^{-}\right]+\Omega\left(C^{+}-C^{-}\right) F+\sum_{\jmath ;: \mathbf{l} \jmath}\left(m_{\imath}-m_{\jmath}\right)\left[\Omega\left(E_{\imath}\right) E_{\jmath}+\Omega\left(E_{\jmath}\right) E_{\imath}\right]
$$


here we have identified the cotangent space of $H^{1,1}(N, \mathbf{R})=H^{2}(N, \mathbf{R})$ with $H_{2}(N, \mathbf{R})$, and each algebraic curve in $\hat{N}$ is used as short-hand for the homology class of its image in $N$. Our goal is thus to show that $\Upsilon$ is never a multiple of $d \mathcal{S}$.

To do this, we begin by considering the case in which $N \neq \hat{N}$, which is to say that at least one of the curves $C^{ \pm}$arises by blowing up an isolated fixed point of the action. By reversing the sign of $\Xi$ if necessary, we may assume that $C^{+}$arises in this way, and let $N^{\prime}$ denote the surface obtained from $\hat{N}$ by contracting $C^{+}$. Because the self-intersection of the image of $F$ in $N^{\prime}$ is $+1, N^{\prime}$ is an iterated blow-up of $\mathbf{C P}_{2}$, and each generic fiber $F$ corresponds to a projective line. Moreover, the image of $C^{-}$in $\mathbf{C P}_{2}$ meets each such projective line in a point, and so $C^{-}$must be the proper transform of a projective line; if $n$ is the number of singular fibers of $\hat{N} \rightarrow \Sigma=\mathbf{C P}_{1}$, we thus have $\left(C^{-}\right)^{2} \leq 1-n$. But plugging

$$
F=\sum_{\text {fiber }} m_{\jmath} E_{\jmath},
$$

$\Omega\left(C^{+}\right)=0$, and $\mathbf{g}=0$ into (5.5), the equation $\mathcal{S}(\Omega)=0$ becomes

$$
0=\Omega\left(C^{-}+2 F+\sum_{\jmath}\left(1-m_{\jmath}\right) E_{\jmath}\right)=\Omega\left(C^{-}+(2-n) F+\sum E_{\jmath}\right),
$$

and hence $n>2$. Thus $\left(C^{-}\right)^{2} \leq 1-n<-1$, so $C^{-}$cannot be a (-1)-curve, and $N^{\prime}=N$. Hence $F \cdot F=1$, and, invoking (5.11),

$$
\begin{aligned}
F \cdot \Upsilon & =F \cdot\left[-C^{-} \Omega(F)-\Omega\left(C^{-}\right) F+\sum_{\imath \mathbf{l}_{\jmath}}\left(m_{\imath}-m_{\jmath}\right)\left[\Omega\left(E_{\imath}\right) E_{\jmath}+\Omega\left(E_{\jmath}\right) E_{\imath}\right]\right] \\
& =-\Omega\left(F+C^{-}-\sum_{\imath}\left(m_{\imath}-1\right) E_{\imath}\right) \\
& =\Omega(F),
\end{aligned}
$$

so that $\Upsilon$ is certainly non-zero, and we need merely show that it is linearly independent from $d \mathcal{S}$.

To do this, let $E_{0}$ be the first curve in some singular fiber, chosen in such a way that $\Omega\left(E_{0}\right)<\Omega(F) / 3$; the latter is possible because the fact that 
$n \geq 3$ tells us that $F$ is homologous to the sum of $C^{-}$and a collection of exceptional curves, at least 3 of which are the first in their respective fibers. Let $E_{1}$ denote the immediate successor of $E_{0}$; the multiplicity of the latter curve is given by $m_{1}=-E_{0}^{2}$, as may be proved by induction. Then

$$
\begin{aligned}
E_{0} \cdot \Upsilon= & E_{0} \cdot\left[-\Omega\left(C^{-}\right) F-\Omega(F) C^{-}+\sum_{\imath \mathbf{l}_{\jmath}}\left(m_{\imath}-m_{\jmath}\right)\left[\Omega\left(E_{\imath}\right) E_{\jmath}+\Omega\left(E_{\jmath}\right) E_{\imath}\right]\right] \\
= & -\Omega(F)+E_{0} \cdot\left[\sum_{0 \mathbf{l}_{\jmath}}\left(1-m_{\jmath}\right) \Omega\left(E_{\jmath}\right) E_{0}\right] \\
& +E_{0} \cdot\left[\sum_{1 \mathbf{l}_{\jmath}}\left(m_{1}-m_{\jmath}\right) \Omega\left(E_{\jmath}\right) E_{1}\right]+E_{0} \cdot\left[\sum_{\imath \mathbf{l} 1}\left(m_{\imath}-m_{1}\right) \Omega\left(E_{\imath}\right) E_{1}\right] \\
= & -\Omega(F)+E_{0}^{2} \sum_{\text {fiber }}\left(1-m_{\jmath}\right) \Omega\left(E_{\jmath}\right)+\sum_{\text {fiber }}\left(-E_{0}^{2}-m_{\jmath}\right) \\
= & -\left(2+E_{0}^{2}\right) \Omega(F)+2\left(1+E_{0}^{2}\right) \Omega\left(E_{0}\right) .
\end{aligned}
$$

On the other hand,

$$
F \cdot d \mathcal{S}=F \cdot\left[C^{-}+2 F+\sum_{\jmath}\left(1-m_{\jmath}\right) E_{\jmath}\right]=3
$$

and

$$
E_{0} \cdot d \mathcal{S}=E_{0} \cdot\left[C^{-}+2 F+\sum_{\jmath}\left(1-m_{\jmath}\right) E_{\jmath}\right]=1+\left(1-m_{1}\right)=2+E_{0}^{2},
$$

so that

$$
\begin{aligned}
\left|\begin{array}{cc}
F \cdot \Upsilon & F \cdot d \mathcal{S} \\
E_{0} \cdot \Upsilon & E_{0} \cdot d \mathcal{S}
\end{array}\right| & =\left|\begin{array}{cc}
\Omega(F) & 3 \\
-\left(2+E_{0}^{2}\right) \Omega(F)+2\left(1+E_{0}^{2}\right) \Omega\left(E_{0}\right) & 2+E_{0}^{2}
\end{array}\right| \\
& =4\left(2+E_{0}^{2}\right) \Omega(F)-6\left(1+E_{0}^{2}\right) \Omega\left(E_{0}\right) .
\end{aligned}
$$

Now this last expression is certainly non-zero if $E_{0}^{2}$ is -1 or -2 ; and if $E_{0}^{2} \leq-3$, the inequality $-\left(E_{0}^{2}+1\right) \Omega\left(E_{0}\right)<-\left(E_{0}^{2}+1\right) \Omega(F) / 3$ yields

$$
\left|\begin{array}{cc}
F \cdot \Upsilon & F \cdot d \mathcal{S} \\
E_{0} \cdot \Upsilon & E_{0} \cdot d \mathcal{S}
\end{array}\right|<2\left(3+E_{0}^{2}\right) \Omega(F) \leq 0 .
$$


Hence $\Upsilon \not \equiv 0 \bmod d \mathcal{S}$ in $H_{2}(N)$, and the claim holds whenever $N \neq \hat{N}$.

We now come to the case in which $N=\hat{N}$. Then, since $F \cdot F=F \cdot E_{\jmath}=0$,

$$
F \cdot d \mathcal{S}=F \cdot\left[C^{+}+C^{-}+2(1-\mathbf{g}) F+\sum_{\jmath}\left(1-m_{\jmath}\right) E_{\jmath}\right]=2,
$$

and

$$
F \cdot \Upsilon=F \cdot\left[\Omega(F)\left[C^{+}-C^{-}\right]+\Omega\left(C^{+}-C^{-}\right) F+\left(E_{\jmath} \text { terms }\right)\right]=0 .
$$

It therefore suffices to show that $\Upsilon \neq 0$ in $H_{2}(N)$. But

$$
C^{+} \cdot \Upsilon=\Omega\left(C^{+}-C^{-}\right)+\left(C^{+}\right)^{2} \Omega(F)+\sum_{\imath} \Omega\left(E_{\imath}\right)
$$

and

$$
C^{-}=\Omega\left(C^{+}-C^{-}\right)-\left(C^{-}\right)^{2} \Omega(F)+\sum_{\jmath}\left(1-m_{\jmath}\right) \Omega\left(E_{\jmath}\right),
$$

so that

$$
\frac{1}{2}\left(C^{+}-C^{-}\right) \cdot \Upsilon=\frac{\left(C^{+}\right)^{2}+\left(C^{-}\right)^{2}}{2} \Omega(F)+\sum_{\jmath}\left(m_{\jmath}-1\right) \Omega\left(E_{\jmath}\right) .
$$

Now $-\left[\left(C^{+}\right)^{2}+\left(C^{-}\right)^{2}\right]$ is precisely the number of times one must blow up along fixed curves of the $\mathbf{C}^{\times}$-action in order to obtain $N$ from a fiber-minimal model; thus $-\left[\left(C^{+}\right)^{2}+\left(C^{-}\right)^{2}\right] \geq n$, where $n$ is the number of singular fibers of $N \rightarrow \Sigma$. If, on the other hand, the first and last exceptional curves of every fiber were to have area $\geq \Omega(F) / 4$, we would have

$$
\sum_{\jmath}\left(m_{\jmath}-1\right)<n\left[\Omega(F)-2 \frac{\Omega(F)}{4}\right]=\frac{n}{2} \Omega(F),
$$

and it would therefore follow that $\frac{1}{2}\left(C^{+}-C^{-}\right) \cdot \Upsilon<\frac{n}{2} \Omega(F)-\frac{n}{2} \Omega(F)=0$, implying $\Upsilon \neq 0$, as desired.

We may therefore assume that either the first or the last curve of some singular fiber has area $<\Omega(F) / 4$; and, by reversing the sign of $\Xi$ if necessary, we may assume that the curve in question is actually the first in its fiber. We thus have an exceptional curve $E_{0}$ which meets $C^{-}$and which satisfies 
$\Omega\left(E_{0}\right)<\Omega(F) / 4$. Now, by essentially the same calculation we used in the $N \neq \hat{N}$ case,

$$
E_{0} \cdot \Upsilon=-\left(2+E_{0}^{2}\right) \Omega(F)+2\left(1+E_{0}^{2}\right) \Omega\left(E_{0}\right),
$$

and in particular $\Upsilon \neq 0$ if $E_{0}^{2}$ is -1 or -2 . If, on the other hand, $E_{0}^{2} \leq-3$, the inequality $\left(1+E_{0}^{2}\right) \Omega\left(E_{0}\right)>\left(1+E_{0}^{2}\right) \Omega(F) / 4$ tells us that

$$
E_{0} \cdot \Upsilon>-\frac{1}{2}\left(E_{0}^{2}+3\right) \Omega(F) \geq 0
$$

Thus $\Upsilon \not \equiv 0 \bmod d \mathcal{S}$, and $H^{2}\left(Z_{N}, \Theta\right)=H^{2}\left(Z_{N}, \Theta_{Z, D \bar{D}}\right)=H^{2}\left(Z, \Theta \otimes \kappa^{-1}\right)=$ 0 , as claimed.

\section{References}

[1] M. Atiyah, N. Hitchin and I. Singer, "Self-Duality in Four Dimensional Riemannian Geometry," Proc. R. Soc. Lond. A 362 (1978) 425-461.

[2] T. Aubin, Equations du Type Monge-Ampère sur les Variétés Kählériennes Compactes, C. R. Acad. Sci. Paris 283A (1976) 119.

[3] C. Boyer, "Conformal Duality and Compact Complex Surfaces," Math. Ann. 274 (1986) 517-526.

[4] W. Barth, C. Peters, and A. Van de Ven, Compact Complex Surfaces, Springer, 1984.

[5] E. Calabi, The Space of Kähler Metrics, Proc. ICM Amsterdam 2 (1954)206-207.

[6] S.K. Donaldson and R.D. Friedman, "Connected Sums of Self-Dual Manifolds and Deformations of Singular Spaces," Nonlinearity 2 (1989) 197-239.

[7] R.D. Friedman, "Global Smoothings of Varieties with Normal Crossings," Ann. Math. 118 (1983) 75-114. 
[8] P. Gauduchon, "Surfaces Kählériennes dont la Courbure Vérifie Certaines Conditions de Positivité," in Géometrie Riemannienne en Dimension 4. Séminaire A. Besse, 1978/1979, (Bérard-Bergery, Berger, and Houzel, eds.), CEDIC/Fernand Nathan, 1981.

[9] R. Godement, "Topologie Algébrique et Théorie des Faisceaux," Hermann, Paris, 1964.

[10] N.J. Hitchin, "Polygons and Gravitons," Math. Proc. Cambridge Phil. Soc. 83 (1979) 465-476.

[11] J. Kim and M. Pontecorvo, "A New Method of Constructing Scalar-Flat Kähler Metrics," preprint, 1993.

[12] C.R. LeBrun, "Explicit Self-Dual Metrics on $\mathbf{C P}_{2} \# \cdots \# \mathbf{C P}_{2}$," J. Diff. Geom. 34 (1991) 223-253.

[13] C.R. LeBrun, "Scalar-Flat Kähler Metrics on Blown-Up Ruled Surfaces," J. reine angew. Math. 420 (1991) 161-177.

[14] C.R. LeBrun, "Self-Dual Manifolds and Hyperbolic Geometry," in Einstein Manifolds and Yang-Mills Connections, (T. Mabuchi and S. Mukai, eds.) Marcel Dekker, 1993, pp. 99-131.

[15] C.R. LeBrun and S.R. Simanca, "On Kähler Surfaces of Constant Positive Scalar Curvature," J. Geom. An. to appear.

[16] C.R. LeBrun and M.A. Singer, "Existence and Deformation Theory for Scalar-Flat Kähler Metrics on Compact Complex Surfaces," Inv. Math. 112 (1993) 273-313.

[17] C.R. LeBrun and M.A. Singer, "A Kummer-type Construction of SelfDual 4-Manifolds," Math. Ann., to appear.

[18] A. Lichnerowicz, "Sur les Transformations Transformations Analytiques des Variétés Kählériennes," C. R. Acad. Sci. Paris 244 (1957) 30113014 . 
[19] A. Nadel, Multiplier Ideal Sheaves and Existence of Kähler-Einstein Metrics of Positive Scalar Curvature, Proc. Natl. Acad. Sci. USA 86 (1989) 7299-7300.

[20] R. Penrose, "Non-linear Gravitons and Curved Twistor Theory," Gen. Rel. Grav. 7 (1976) 31-52.

[21] M. Pontecorvo, "On Twistor Spaces of Anti-Self-Dual Hermitian Surfaces," Trans. Am. Math. Soc. 331 (1992) 653-661.

[22] Z. Ran, "Deformations of Maps," Lecture Notes in Mathematics 1389 (1989) 246-253.

[23] Z. Ran, "Stability of Certain Holomorphic Maps" J. Diff. Geom. 34 (1991), 37-47.

[24] I. Satake, "The Gauss-Bonnet Theorem for V-manifolds," J. Math. Soc. Japan 9 (1957) 464-476.

[25] Y.T. Siu, The Existence of Kähler-Einstein Metrics on Manifolds with Positive Anti-Canonical Line Bundle and Suitable Finite Symmetry Group, Ann. Math. 127 (1988) 585-627.

[26] C.H. Taubes, "The Existence of Anti-Self-Dual Metrics," J. Diff. Geometry 36 (1992) 163-253.

[27] G. Tian, On Calabi's Conjecture for Complex Surfaces with Positive First Chern Class, Inv. Math. 101 (1990) 101-172.

[28] G. Tian and S.T. Yau, Kähler-Einstein Metrics on Complex Surfaces with $\mathbf{c}_{1}>$ 0, Comm. Math. Phys. 112 (1987) 175-203.

[29] S.T. Yau, "On the Curvature of Compact Hermitian Manifolds," Inv. Math. 25 (1974) 213-239.

[30] S.T. Yau, "On the Ricci-Curvature of a Complex Kähler Manifold and the Complex Monge-Ampère Equations," Comment. Pure Appl. Math. 31 (1978) 339-411. 